\title{
ASYMPTOTIC BEHAVIOR OF ACOUSTIC WAVES SCATTERED BY VERY SMALL OBSTACLES
}

\author{
Hélène BarucQ ${ }^{1}$, Julien Diaz ${ }^{1}$, Vanessa Mattesi ${ }^{2}$ and Sebastien Tordeux ${ }^{1, *}$
}

\begin{abstract}
The direct numerical simulation of the acoustic wave scattering created by very small obstacles is very expensive, especially in three dimensions and even more so in time domain. The use of asymptotic models is very efficient and the purpose of this work is to provide a rigorous justification of a new asymptotic model for low-cost numerical simulations. This model is based on asymptotic near-field and far-field developments that are then matched by a key procedure that we describe and demonstrate. We show that it is enough to focus on the regular part of the wave field to rigorously establish the complete asymptotic expansion. For that purpose, we provide an error estimate which is set in the whole space, including the transition region separating the near-field from the far-field area. The proof of convergence is established through Kondratiev's seminal work on the Laplace equation and involves the Mellin transform. Numerical experiments including multiple scattering illustrate the efficiency of the resulting numerical method by delivering some comparisons with solutions computed with a finite element software.
\end{abstract}

Mathematics Subject Classification. 35C20, 35L05, 74J20.

Received April 3, 2020. Accepted July 5, 2020.

\section{General introduction}

Mechanical wave simulations are of great interest in many applications due to their capability of transporting information in the medium they propagate into. In particular, they are capable of detecting very small heterogeneities that we can visualize using the recording of the scattered fields generated whenever the wave field encounters obstacles $[8,28-30]$. This phenomenon of multiple diffraction can be reproduced numerically and for precise calculations, the finite element method is very efficient. Indeed, finite element methods are able to capture the characteristics of the obstacles including their shape by the use of tetrahedral meshes. However, if the obstacles are very small, their use can lead to very high computational costs, especially because it is necessary to mesh very finely in the neighborhood of the obstacles. This is a considerable drawback which motivates a clear interest in the development of numerical methods which avoid the use of refined meshes. It should also be noted that in addition to high direct computational costs, the design of unstructured meshes can also be difficult and very time consuming and also contributes to high implementation costs.

Keywords and phrases. Acoustic wave propagation, matched asymptotic expansion method, scattering problem, Mellin transform, singularity theory.

1 Magique 3D, INRIA, E2S-UPPA, LMAP UMR CNRS 5142, Pau, France.

2 Department of Electrical Engineering and Computer Science, University of Liège, Liège, Belgium.

*Corresponding author: sebastien.tordeux@inria.fr, sebastien.tordeux@gmail.com 
Most of the time, the use of explicit time schemes is preferred to integrate wave equations. This choice is dictated by the need of avoiding inversion of the mass matrix at each time iteration, in order to limit computational costs as well as memory usage. In this case, the explicit schemes are not fully suitable for direct numerical simulation. Indeed, they are stable only by respecting the Courant-Friedrichs-Levy condition which shows a linear dependency of the time step with the space step. Globally applying this condition to a direct numerical simulation based on a locally refined grid leads to increased computational costs since the time step will be adapted to the smallest cell. This is particularly disabling when the domain has only a few small obstacles. And even if the domain contains a lot of them, it should not be forgotten that explicit time schemes can generate numerical pollution mainly due to dispersion effects caused by the application of a global time step which is only adapted to the smallest cells. This has motivated the development of time-explicit schemes using local time steps that can be locally adapted to space steps (see e.g. $[16,38]$.) Local time stepping methods are particularly interesting when the number of small cells is high. When this is not the case, it may be more efficient to use locally implicit schemes (see e.g. [34]). These schemes result from coupling an unconditionally stable implicit scheme applied in the areas of the mesh composed of small cells with an explicit scheme elsewhere. One can also take another side and decide to work on the mathematical model itself in order to keep an explicit scheme for time integration. The work to be done on the equations then consists in erasing, as it was, the small obstacles so that they do not have to be taken into account by the mesh. The latter will no longer contain small cells and we will thus be able to integrate the equations with a larger time step. To build such a mathematical model, asymptotic methods turn out to be very efficient besides having a rigorous framework for establishing convergence results which ensure that the solution calculated by solving the asymptotic model converges towards the solution of the initial problem. Asymptotic methods have been applied several times to stationary problems (see $[13,21,23,26,40,42])$ and to the best of our knowledge, Mattesi and Tordeux $[32,33]$ represents the first attempt in the time domain.

The construction of a reduced model for representing scattering problems relies on a key procedure which consists in matching the asymptotic expansions of the near and far field in order to get a full representation of the scattered field. We describe that procedure in the following section. This work includes two new contributions in addition to being carried out in the time domain and in three-dimensional space. On the one hand, we extend the computational method proposed in $[32,33]$ to the case of several small obstacles. On the other hand, we develop a mathematical analysis which justifies the asymptotic model proposed in $[32,33]$ for the case of a single small obstacle.

The paper is organized as follows. We begin with describing the matching procedure for the construction of the asymptotic model. This requires defining the near-field and far-field approximations which are then matched to give a representation of the diffracted field throughout the space, disregarding the obstacle which should no longer be taken into account in the computational method. Then we extend the asymptotic model proposed to the case of scattering by multiple small obstacles. We illustrate the interest of our approach by carrying out several numerical experiments which consist in solving the problem of multiple scattering by using either the asymptotic model or the complete model. The latter is solved with a discontinuous finite element method. All the calculations we have performed show that the numerical solution obtained from the asymptotic model achieves a level of precision comparable to that obtained with the finite element method when we consider small obstacles. In addition, as expected, the computational costs are clearly to the advantage of the asymptotic method, which moreover does not require any mesh and thus avoiding a step that can be difficult and often time-consuming. For example, in the case of 216 obstacles, the calculations on the reduced model take $2.56 \mathrm{~s}$ while the direct simulation lasts $12 \mathrm{~h}$ for a computation carried out in parallel on 576 processors. We finish our study by developing a mathematical analysis to rigorously justify the proposed reduced model. The analysis consists in establishing a convergence result which uses the Mellin transform (see [7]) in the formalism of Kondratiev spaces [24]. 


\section{Description of the MATCHing of ASYMPtotic EXPANSiOns}

To simplify the introduction of the matching method, we limit ourselves to the case of a single obstacle defined as the small sphere $B_{\varepsilon}$ with a near-zero radius $\varepsilon$. We will generalize the method to the case of several obstacles by the end of the paper. The corresponding scattering problem posed in $\Omega=\mathbb{R}^{3} \backslash B_{\varepsilon}$ reads as:

$$
\begin{cases}\Delta u_{\varepsilon}(\mathbf{x}, t)-\frac{1}{c^{2}} \partial_{t}^{2} u_{\varepsilon}(\mathbf{x}, t)=0, & \text { on } \Omega, \quad \text { on } \partial \Omega, \\ u_{\varepsilon}(\mathbf{x}, t)=0, & \text { on } \Omega, \\ u_{\varepsilon}(\mathbf{x}, 0)=v_{0}(\mathbf{x}), \quad \partial_{t} u_{\varepsilon}(\mathbf{x}, 0)=v_{1}(\mathbf{x}),\end{cases}
$$

where $\mathbf{x}=(x, y, z)$ is the spatial variable defined in $\mathbb{R}^{3}, t \geq 0$ denotes the time variable and $\Delta=\partial_{x}^{2}+\partial_{y}^{2}+\partial_{z}^{2}$ is the Laplace operator in the cartesian coordinate system.

Hypothesis 2.1. The initial data $v_{0}, v_{1}: \mathbb{R}^{3} \longrightarrow \mathbb{R}$ are of class $C^{\infty}$ and their support is included in $B_{r_{\star}} \backslash\{0\}$ with $B_{r_{\star}}:=\left\{\mathbf{x} \in \mathbb{R}^{3}:|\mathbf{x}| \leq r_{\star}\right\}, r_{\star}$ being a non negative real number with $\varepsilon \ll r_{\star}$.

\subsection{Asymptotic parameterization}

The construction of the approximation of the diffracted field involves a certain number of physical parameters which will intervene in the characterization of the wave field. First of all, we introduce the parameter $\varepsilon$ which defines the radius of the obstacle. It is small in the sense that it is very small in front of the mean wavelength $l_{m}$ of the wave field. The latter is defined as the distance between two maximum values of the Fourier transform of the signal. The Fourier transform is most of the time centered around a characteristic angular frequency $\omega_{m}$ which defines a characteristic wavelength $l_{m}=\frac{c}{\omega_{m}}$ where $c$ denotes the propagation velocity of the wave. By small obstacle, we then mean that $\varepsilon$ is very small in front of $l_{m}$.

Now that the asymptotic parameters are defined, we move on the parameterization of the exterior domain surrounding the scatterer. For any $\mathbf{x}$ in the exterior of $B_{\varepsilon}$, we set:

$$
\mathbf{x}=\varepsilon^{s} l_{m}^{1-s} \mathbf{X}, s \in(0,1),|\mathbf{X}| \geq 1 .
$$

Then we have:

- if $s=0, \mathbf{x}=l_{m} \mathbf{X} ; \mathbf{x}$ is located in the so-called far field region where the propagation phenomenon is preponderant while the obstacle has little impact on the scattered field. The scatterer acts like a sourcepoint so that the scattered field can be represented by an asymptotic expansion set in $R^{3}$.

- if $s=1, \mathbf{x}=\varepsilon \mathbf{X} ; \mathbf{x}$ is located in the so-called near-field region where the obstacle has strong impact on the scattered field while the propagation phenomenon is negligible. A quasi-static behavior of the scattered field is actually expected from its asymptotic expansion.

- if $0<s<1, \mathbf{x}$ is in the so-called matching area where both the propagation and the obstacle effects have to be taken into account. Hence this is here where the far field expansion has to match the near-field one to ensure that the final asymptotic representation provides a reliable representation of the scattered field. For this, it is crucial to see that if $\mathbf{x}$ is of order $\varepsilon^{s} \ell_{m}^{1-s}$ then $\mathbf{x} / \varepsilon$ is of order $\varepsilon^{s-1} \ell_{m}^{1-s}$. The first one tends to zero as $\varepsilon$ tends to zero whereas the second tends towards infinity.

Remark 2.2. It is worth noting that it is $\varepsilon$ which acts as the small parameter. Hence in the following, the scattered field will be given as a series involving powers of $\varepsilon$.

\subsection{The far-field approximation}

In the far-field region, we seek a solution $u_{\varepsilon}$ to problem (2.1) in the following form (Fig. 1):

$$
u_{\varepsilon}(\mathbf{x}, t) \sim \sum_{i=0}^{+\infty} u_{i}(\mathbf{x}, t) \varepsilon^{i},
$$




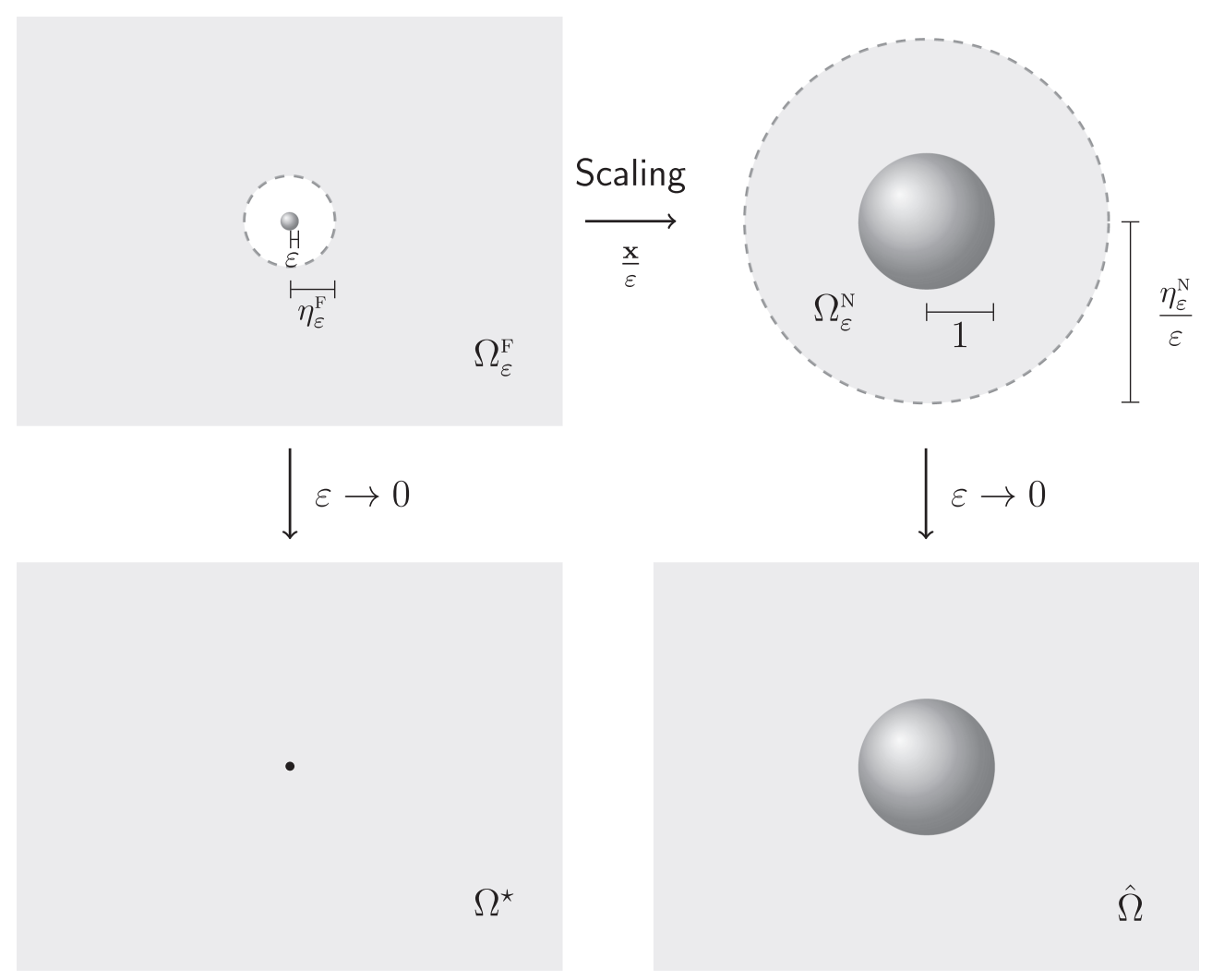

FiguRE 1. Far-field and near-field domains $\Omega_{\varepsilon}^{\mathrm{F}}$ and $\Omega_{\delta}^{\mathrm{N}}$ and their limit $\Omega^{\star}$ and $\hat{\Omega}$.

where each term $u_{i}: \mathbb{R}^{3, *} \times \mathbb{R}^{+} \longrightarrow \mathbb{R}$ is a space-time function defined in the domain $\mathbb{R}^{3} \backslash\{0\}$. Following the methodology described in [9], we have:

- The first term $u_{0}$ is $C^{\infty}$ and is defined as a solution to the wave equation set in the whole space (i.e. without obstacle)

$$
\begin{cases}\Delta u_{0}(\mathbf{x}, t)-\frac{1}{c^{2}} \partial_{t}^{2} u_{0}(\mathbf{x}, t)=0, & \text { on } \quad \mathbb{R}^{3}, \quad \forall t \geq 0, \\ u_{0}(\mathbf{x}, 0)=v_{0}(\mathbf{x}), \quad \partial_{t} u_{0}(\mathbf{x}, 0)=v_{1}(\mathbf{x}), & \text { on } \quad \mathbb{R}^{3} .\end{cases}
$$

The function $u_{0}$ actually represents the regular part of the wave field and is given thanks to the theory of separation of variables as

$$
u_{0}(\mathbf{x}, t)=\sum_{n=0}^{+\infty} \sum_{m=-n}^{n} u_{m, n}(r, t) Z_{m, n}(\theta, \varphi)
$$

The functions $Z_{m, n}$ are the spherical angular harmonics and are given by

$$
Z_{m, n}(\theta, \varphi)=\frac{1}{\sqrt{2 \pi}} \overline{P_{n}^{|m|}}(\cos \theta) \exp (i m \varphi)
$$

where $\overline{P_{n}^{m}}$ are the normalized Legendre function $\left(\left\|P_{n}^{m}\right\|_{L^{2}([-1,1])}=1\right)$ of degree $n \in \mathbb{N}$ and of order $m \in[0, n]$. It is defined for $x \in[-1,1]$ by

$$
\overline{P_{n}^{m}}(x)=\sqrt{\left(n+\frac{1}{2}\right) \frac{(n-m) !}{(n+m) !}} \times\left(1-x^{2}\right)^{\frac{m}{2}} d_{x}^{m} P_{n}(x),
$$


where $P_{n}$ is the Legendre polynomial of degree $n$ given by

$$
P_{n}(x)=\frac{1}{2^{n} n !} d_{x}^{n}\left[\left(x^{2}-1\right)^{n}\right] .
$$

More details on Legendre polynomials are available for instance in ([37], see p. 35), in ([21], see p. 47) or in ([19], see p. 353). The radial function $u_{m, n}$ has the following expression

$$
u_{m, n}(r, t)=R_{m, n}\left(\Lambda_{0, m, n} r, t\right)
$$

where $\Lambda_{0, m, n}: \mathbb{R} \longrightarrow \mathbb{R}$ is the amplitude of the regular mode $R_{m, n}$ which takes the form

$$
R_{m, n}(\Lambda, r, t)=\sum_{\ell=0}^{n} q_{n}^{\ell} \frac{\Lambda^{(n-\ell)}(t-r / c)-(-1)^{n-\ell} \Lambda^{(n-\ell)}(t+r / c)}{4 \pi r^{\ell+1} c^{n-\ell}} .
$$

- For $i>0$, the far-field terms $u_{i}$ are singular solutions to the wave equation which are undefined at the origin and satisfy

$$
\left\{\begin{array}{lll}
\Delta u_{i}(\mathbf{x}, t)-\frac{1}{c^{2}} \partial_{t}^{2} u_{i}(\mathbf{x}, t)=0, & \forall \mathbf{x} \in \mathbb{R}^{3, *}, & \forall t \geq 0, \\
u_{i}(\mathbf{x}, 0)=0, & \partial_{t} u_{i}(\mathbf{x}, 0)=0, & \forall \mathbf{x} \in \mathbb{R}^{3, *} .
\end{array}\right.
$$

We adopt the notation $\mathbb{R}^{3, *}$ to refer to the space $\mathbb{R}^{3}$ free of origin. Each term $u_{i}$ is represented as

$$
u_{i}(\mathbf{x}, t)=\sum_{n=0}^{+\infty} \sum_{m=-n}^{n} M_{m, n}\left(\Lambda_{i, m, n} ; r, t\right) Z_{m, n}(\theta, \varphi),
$$

which is a finite sum of multipoles $M_{m, n}(\Lambda ; \cdot): \mathbb{R}^{+, *} \times \mathbb{R} \longrightarrow \mathbb{R}$, where $\mathbb{R}^{+, *}$ refers to non negative real numbers, defined as functions of their magnitude $\Lambda_{i, m, n}: \mathbb{R} \longrightarrow \mathbb{R}$

$$
M_{m, n}\left(\Lambda_{i, m, n} ; r, t\right)=\left(\sum_{\ell=0}^{n} q_{n}^{\ell} \frac{\Lambda_{i, m, n}^{(n-\ell)}(t-r / c)}{4 \pi r^{\ell+1} c^{n-\ell}}\right) .
$$

We have $\Lambda_{i, m, n}(t-r / c)=0$ for $t-r / c \leq 0$ and $q_{n}^{\ell}=\frac{(n+\ell) !}{\ell !(n-\ell) !} \frac{1}{2^{\ell}}$, see [32,33].

\subsection{The near-field approximation}

Here we use the dimensionless variable $\mathbf{X}=\frac{\mathbf{x}}{\varepsilon}$ which satisfies $R:=|\mathbf{X}| \geq 1$ and we seek for $U_{\varepsilon}(\mathbf{X}, t)$ which satisfies

$$
U_{\varepsilon}(\mathbf{X}, t)=u_{\varepsilon}(\mathbf{x}, t)
$$

and

$$
U_{\varepsilon}(\mathbf{X}, t) \sim \sum_{i=0}^{+\infty} U_{i}(\mathbf{X}, t) \varepsilon^{i} .
$$

Given that $\Delta_{\mathbf{x}}=\frac{1}{\varepsilon^{2}} \Delta_{X}$, if we have $\Delta_{\mathbf{x}} u_{\varepsilon}-\frac{\partial_{t}^{2}}{c^{2}} u_{\varepsilon}=0$ we also have $\frac{1}{\varepsilon^{2}} \Delta U_{\varepsilon}(\mathbf{X}, t)=\frac{\partial_{t}^{2}}{c^{2}} U_{\varepsilon}(\mathbf{X}, t)$. Using $(2.15)$, we formally get

$$
\frac{1}{\varepsilon^{2}} \Delta U_{0}+\frac{1}{\varepsilon} \Delta U_{1}+\sum_{i=0}^{+\infty} \varepsilon^{i}\left(\Delta U_{i+2}(\mathbf{X}, t)\right)=\sum_{i=0}^{+\infty} \varepsilon^{i}\left(\frac{\partial_{t}^{2}}{c^{2}} U_{i}(\mathbf{X}, t)\right) .
$$

Then, by identifying each term multiplied by $\varepsilon^{i}$ with $i \geq-2$, we get that the two first terms $U_{0}$ and $U_{1}$ in $(2.16)$ solve the Laplace equation

$$
\Delta U_{0}(\mathbf{X}, t)=\Delta U_{1}(\mathbf{X}, t)=0 \text { for } R \geq 1
$$


whereas $U_{i}$, for $i \geq 2$, satisfies the nested Laplace equations given by

$$
\Delta U_{i}(\mathbf{X}, t)=\frac{\partial_{t}^{2}}{c^{2}} U_{i-2}(\mathbf{X}, t) \text { for } R \geq 1 .
$$

Moreover all the terms of this expansion including those for $i=0$ and $i=1$, solve the Dirichlet boundary condition

$$
U_{i}(\mathbf{X}, t)=0 \text { for } R=1 .
$$

\subsection{The matching procedure}

To make the asymptotic representation of the scattered field self-content, we have to realize the matching between both developments previously introduced. For that purpose, we go back to the relation that links the variables $\mathbf{x}$ and $\mathbf{X}$. Given that $\mathbf{x}=\varepsilon \mathbf{X}$, we have that $\mathbf{x}$ tends to be close to 0 while $\mathbf{X}$ tends to infinity. This leads us to consider an expansion of each $u_{i}$ in the vicinity of the origin as well as a development of $U_{i}$ in a neighborhood of infinity. For this, we use the spherical coordinate system and write down:

$$
u_{i}(r, \theta, \varphi, t) \sim \sum_{p=-\infty}^{+\infty} u_{i, p}(\theta, \varphi, t) r^{p}
$$

for $u_{i}$ and

$$
U_{i}(R, \theta, \varphi, t) \sim \sum_{p=-\infty}^{+\infty} U_{i, p}(\theta, \varphi, t) R^{p}, \quad \text { with } R=\frac{r}{\varepsilon},
$$

for $U_{i}$. Identifying $u_{\varepsilon}$ and $U_{\varepsilon}$ in the matching zone and using the different expansions (2.3), (2.15), (2.20) and (2.21), we obtain the following matching relation

$$
u_{i, p}(\theta, \varphi, t)=U_{i+p, p}(\theta, \varphi, t) \quad \forall i \in \mathbb{Z}, \quad p \in \mathbb{Z},
$$

or equivalently

$$
U_{i, p}(\theta, \varphi, t)=u_{i-p, p}(\theta, \varphi, t) \quad \forall i \in \mathbb{Z}, \quad p \in \mathbb{Z} .
$$

In the following, we adopt the convention

$$
u_{i} \equiv 0 \quad \text { and } \quad U_{i} \equiv 0 \quad \text { for } i<0
$$

which implies in particular that for $i \geq 0$

$$
u_{i, p}(\theta, \varphi, t)=0 \text { for } p<-i \quad \text { and } \quad U_{i, p}(\theta, \varphi, t)=0 \text { for } p>i .
$$

Expansions (2.20) and (2.21) read then

$$
\left\{\begin{array}{l}
u_{i}(r, \theta, \varphi, t) \sim \sum_{p=-i}^{+\infty} u_{i, p}(\theta, \varphi, t) r^{p}, \\
U_{i}(R, \theta, \varphi, t) \sim \sum_{p=-\infty}^{i} U_{i, p}(\theta, \varphi, t) R^{p}, \quad \text { with } R=\frac{r}{\varepsilon}
\end{array}\right.
$$

Now we move on to the explicit writing of the first terms of (2.26). In the vicinity of the origin, the far-field term $u_{0}$ is a regular solution to the wave equation (2.4) and we have

$$
u_{0}(\mathbf{x}, t)=u_{0}(\mathbf{0}, t)+\nabla u_{0}(\mathbf{0}, t) \cdot \mathbf{x}+\underset{r \rightarrow 0}{O}\left(r^{2}\right) .
$$


By identifying the different terms, we get that

$$
u_{0,0}(\theta, \varphi, t)=u_{0}(\mathbf{0}, t) \text { and } u_{0,1}(\theta, \varphi, t)=\nabla u_{0}(\mathbf{0}, t) \cdot \frac{\mathbf{x}}{r} .
$$

The matching conditions (2.23) and (2.25) then imply that

$$
U_{0,0}(\theta, \varphi, t)=u_{0,0}(\theta, \varphi, t)=u_{0}(\mathbf{0}, t) \text { and } U_{0, p}(\theta, \varphi, t)=0 \quad \text { for } \quad p>0 .
$$

In particular,

$$
U_{0}(\mathbf{X}, t)=u_{0}(\mathbf{0}, t)+\underset{r \rightarrow 0}{O}(r) .
$$

Regarding $U_{0}$, it is a solution to the Laplace equation (2.17) which admits the following modal expansion [35]

$$
U_{0}(R, \theta, \varphi)=\sum_{n=0}^{+\infty}\left(A_{n} R^{n}+B_{n} R^{-n-1}\right) Z_{m, n}(\theta, \varphi) .
$$

Taking (2.28) into account and given that $Z_{0,0}=\frac{1}{\sqrt{4 \pi}}$, we deduce that $A_{0}=\sqrt{4 \pi} u_{0}(\mathbf{0}, t)$ and $A_{n}=0$ for $n \geq 1$. It follows from (2.19), that $B_{n}=-A_{n}$. We then get

$$
U_{0}(\mathbf{X}, t)=u_{0}(\mathbf{0}, t)\left(1-\frac{1}{R}\right),
$$

and the matching condition (2.23) implies that

$$
U_{0,-1}(\theta, \varphi, t)=-u_{0}(\mathbf{0}, t) \text { and } U_{0,-2}(\theta, \varphi, t)=0 .
$$

Thanks to $(2.22)$, we have $u_{1,-1}(\theta, \varphi, t)=U_{0,-1}(\theta, \varphi, t)=-u_{0}(\mathbf{0}, t)$. It follows from $(2.25)$ that $u_{1, p}(\theta, \varphi, t)=0$ for $p<-1$. This implies that

$$
u_{1}(\mathbf{x}, t)=-\frac{u_{0}(\mathbf{0}, t)}{r}+\underset{r \rightarrow 0}{O}(1) .
$$

Next, we compare (2.31) with the term $u_{1}$ in (2.12). It is worth noting that the term $M_{m, n}\left(\Lambda_{1, m, n}, t\right)$ in $(2.12)$ is computed in the vicinity of $\mathbf{x}=0$ according to the representation formula:

$$
M_{m, n}\left(\Lambda_{1, m, n} ; \mathbf{x}, t\right)=\frac{q_{n}^{n} \Lambda_{1, m, n}(t)}{4 \pi r^{n+1}} Z_{m, n}(\theta, \varphi)+\underset{r \rightarrow 0}{O}\left(\frac{1}{r^{n}}\right) .
$$

Then, remarking that $\Lambda_{1, m, n} \equiv 0$, barring $(m, n)=(0,0)$, we get

$$
u_{1}(\mathbf{x}, t)=M_{0,0}\left(\Lambda_{1,0,0} ; \mathbf{x}, t\right)=\frac{\Lambda_{1,0,0}\left(t-\frac{r}{c}\right)}{(4 \pi)^{\frac{3}{2}}} \frac{1}{r}=\underbrace{\frac{\Lambda_{1,0,0}(t)}{(4 \pi)^{\frac{3}{2}}}}_{u_{1,-1}(\theta, \varphi, t)} \frac{1}{r}-\underbrace{\frac{\Lambda_{1,0,0}^{\prime}(t)}{(4 \pi)^{\frac{3}{2}} c}}_{u_{1,0}(\theta, \varphi, t)}+\underset{r \rightarrow 0}{O}(r) .
$$

It follows that $\Lambda_{1,0,0}(t)=-(4 \pi)^{3 / 2} u_{0}(\mathbf{0}, t)$ and consequently we obtain

$$
u_{1}(\mathbf{x}, t)=-\frac{u_{0}\left(\mathbf{0}, t-\frac{r}{c}\right)}{r} \quad \text { and } \quad u_{1,0}(\theta, \varphi, t)=\frac{\partial_{t} u_{0}(\mathbf{0}, t)}{c} .
$$

Similarly, we get the first order near-field term

$$
U_{1}(\mathbf{X}, t)=\frac{\partial_{t} u_{0}(\mathbf{0}, t)}{c}\left(1-\frac{1}{R}\right)+\nabla u_{0}(\mathbf{0}, t) \cdot\left(\mathbf{X}-\frac{\mathbf{X}}{R^{3}}\right),
$$


while the second order far-field term is given by

$$
u_{2}(\mathbf{x}, t)=-\frac{\partial_{t} u_{0}\left(\mathbf{0}, t-\frac{r}{c}\right)}{r c} .
$$

We then end up with the second order far-field expansion:

$$
\left\{\begin{aligned}
u_{\varepsilon, 2}(\mathbf{x}, t) & :=u_{0}(\mathbf{x}, t)+\varepsilon u_{1}(\mathbf{x}, t)+\varepsilon^{2} u_{2}(\mathbf{x}, t) \\
& =u_{0}(\mathbf{x}, t)-\varepsilon \frac{u_{0}\left(\mathbf{0}, t-\frac{r}{c}\right)}{r}-\varepsilon^{2} \frac{\partial_{t} u_{0}\left(\mathbf{0}, t-\frac{r}{c}\right)}{r c},
\end{aligned}\right.
$$

where $u_{0}$ is the regular solution to the wave equation in the whole space (2.4) (i.e. without obstacle).

Moreover, we have

$$
u_{0}\left(\mathbf{0}, t-\frac{r-\varepsilon}{c}\right)=u_{0}\left(\mathbf{0}, t-\frac{r}{c}\right)+\frac{\varepsilon}{c} \partial_{t} u_{0}\left(\mathbf{0}, t-\frac{r}{c}\right)+\underset{\varepsilon \rightarrow 0}{O}\left(\varepsilon^{2}\right) .
$$

This last expression can be injected in (2.37) to get a second order approximation of the wave field:

Theorem 2.3. A second order far-field approximation of the exact solution is given by

$$
\left.u_{\varepsilon, 2}^{\bmod }(\mathbf{x}, t):=u_{0}(\mathbf{x}, t)-\varepsilon \frac{u_{0}\left(\mathbf{0}, t-\frac{r-\varepsilon}{c}\right)}{r}\right] .
$$

Remark 2.4. This formal result can be made rigorous by an error analysis, [40]. Given that $\left(u_{\varepsilon}=u_{\varepsilon, 2}+O\left(\varepsilon^{3}\right)\right)$ and $u_{\varepsilon, 2}=u_{\varepsilon, 2}^{\text {mod }}+O\left(\varepsilon^{3}\right)$, we obtain that for all $\mathbf{x} \neq 0$, there exist $C_{\mathbf{x}}>0$ and $\varepsilon_{\mathbf{x}}>0$ such that for all $\left.\varepsilon \in\right] 0, \varepsilon_{\mathbf{x}}[$.

$$
\left|u_{\varepsilon}(\mathbf{x}, t)-u_{\varepsilon, 2}^{\bmod }(\mathbf{x}, t)\right| \leq C \varepsilon^{3} .
$$

It is worth noting that (2.37) and (2.39) do not involve the obstacle, which explains why using such representation of the scattered field will not require considering the obstacle as a geometrical object. By this way, there will be no need to reproduce the surface of the scatterer by a set of points, the latter being modeled as a point source.

\section{Application to MUltiple SCATtering}

We consider the scattering problem (2.1) created by $N$ spheres of radius $\varepsilon$ and center $\mathbf{x}_{n} \in \mathbb{R}^{3}, 1 \leq n \leq N$. The propagation domain is denoted by $\Omega=\mathbb{R}^{3} \backslash\left(\bigcup_{n=1}^{N} B_{\varepsilon}\left(\mathbf{x}_{n}\right)\right)$. The total field $u_{\varepsilon}$ is decomposed as the sum of an incident field $u_{0}$ defined by (2.4) and a finite superposition of scattered fields $u_{n}$ :

$$
u_{\varepsilon}(\mathbf{x}, t)=u_{0}(\mathbf{x}, t)+\sum_{n=1}^{N} u_{n}(\mathbf{x}, t) .
$$

There are different ways for computing an approximation of the solution of this problem. The field $u_{n}$ can be approximated by a mono-polar source of amplitude $\Lambda_{n}$ in the following way:

$$
u_{n}(\mathbf{x}, t)=\frac{\Lambda_{n}\left(t-\frac{r}{c}\right)}{4 \pi\left|\mathbf{x}-\mathbf{x}_{n}\right|} \quad \text { with } \Lambda_{n}(t)=-4 \pi \varepsilon u_{\text {inc }, n}\left(\mathbf{x}_{n}, t+\frac{\varepsilon}{c}\right)
$$

where $u_{\text {inc,n }}$ is the field illuminating the obstacle $B_{\varepsilon}\left(\mathbf{x}_{n}\right)$. Then, the approximate solution depends on the definition of $u_{\text {inc }, n}$. Here, we address the following possibilities: 
- Adopting the first order Born approximation, the interactions between obstacles are neglected. In that case,

$$
u_{\text {inc }, n}(\mathbf{x}, t)=u_{0}(\mathbf{x}, t)
$$

This leads to an explicit formula of $u_{n}$ from:

$$
\Lambda_{n}(t)=-4 \pi \varepsilon u_{0}\left(\mathbf{x}_{n}, t+\frac{\varepsilon}{c}\right) .
$$

- The Foldy-Lax model, written classically for harmonic waves [9,11,12,18,31], includes the interactions between obstacles. Here for a given obstacle $B_{\varepsilon}\left(\mathbf{x}_{n}\right)$, the incident field is the sum of the incident wave $u_{0}$ and the fields scattered by all the other obstacles:

$$
u_{\text {inc }, n}(\mathbf{x}, t)=u_{0}(\mathbf{x}, t)+\sum_{m=1, m \neq n}^{N} u_{m}(\mathbf{x}, t)
$$

with the corresponding amplitude computed according to the definition (3.2)

$$
\Lambda_{n}(t)=-4 \pi \varepsilon\left(u_{0}\left(\mathbf{x}_{n}, t+\frac{\varepsilon}{c}\right)+\sum_{m=1, m \neq n}^{N} \frac{\Lambda_{m}\left(t-\frac{d_{n, m}-\varepsilon}{c}\right)}{4 \pi d_{n, m}}\right) \quad \text { with } d_{n, m}=\left|\mathbf{x}_{n}-\mathbf{x}_{m}\right| .
$$

Numerically, the unknown functions $t \mapsto \Lambda_{n}(t)$ will be approximated on a regular grid in time: $t_{k}=k \delta t$ with $k \in \mathbb{N}$. In the following, we denote by $\left(\Lambda_{n}\right)_{k}$ the numerical approximation of $\Lambda_{n}\left(t_{k}\right)$. It is worth noting that the numerical solution of system (3.5) requires some evaluations at time $t_{k}-\frac{d_{n, m}-\varepsilon}{c}$ for all $n \neq m$. These are discrete times which may not be on the time grid. An interpolation technique is then used to evaluate the right hand side with respect to the values on the grid.

$$
\left(\Lambda_{n}\right)_{k}=-4 \pi \varepsilon\left(u_{0}\left(\mathbf{x}_{n}, t_{k}+\frac{\varepsilon}{c}\right)+\sum_{m=1, m \neq n}^{N} \frac{\widetilde{\Lambda}_{m}\left(t_{k}-\frac{d_{n, m}-\varepsilon}{c}\right)}{4 \pi d_{n, m}}\right) .
$$

In the above expression, $\widetilde{\Lambda}_{m}$ is an interpolation of $\Lambda_{m}$ given by

$$
\left\{\begin{array}{l}
\widetilde{\Lambda}_{m}(t)=0 \text { for } t<0 \\
\widetilde{\Lambda}_{m}(t)=\left(\Lambda_{m}\right)_{k} \frac{t_{k+1}-t}{\delta t}+\left(\Lambda_{m}\right)_{k+1} \frac{t-t_{k}}{\delta t} \text { with } t_{k} \leq t<t_{k+1}
\end{array}\right.
$$

In the following numerical study, we will compare results obtained with

- The Born approximation.

- The Foldy Lax model.

- A Direct Numerical Simulation performed with the numerical Library Hou10ni [6]. This software package is able to simulate time-dependent acoustic wave propagation in $3 \mathrm{D}$. The space discretization is based upon the Interior Penalty Discontinuous Galerkin method $[2,4,5,15,20]$ and the time integration is carried out with a Leap Frog scheme. There is a need in truncating the computational domain and for this, we use a first order absorbing boundary condition which is set on the exterior boundary. The code integrates the $p$-adaptivity option, which allows to use different orders of approximation ranging from $p=1$ to $p=6$. In this way, the computational costs of the direct simulation method are minimized by adopting a refined mesh in the vicinity of obstacles and larger cells elsewhere. 
In each numerical experiment, we consider an incident wave which is analytically defined by

$$
u_{0}(\mathbf{x}, t)=\frac{\Lambda_{0}\left(t-\frac{|\mathbf{x}|}{c}\right)}{4 \pi|\mathbf{x}|}
$$

with

$$
\Lambda_{0}(t)=\left(t-t_{0}\right) e^{-\alpha_{0}^{2}\left(t-t_{0}\right)^{2}}
$$

The wave speed $c$ is equal to 1 . The real numbers $\alpha_{0}=5$ is a characteristic frequency whereas $t_{0}=\frac{1.2 \pi}{\alpha_{0}}$ is a characteristic time. Most of the energy of the signal corresponds to the wave length:

$$
\lambda \in\left[\lambda_{m},+\infty\left[\quad \text { with } \lambda_{m}=c t_{0} \simeq 0.75 .\right.\right.
$$

First numerical experiment: the case of five spheres. We consider two configurations with five spherical obstacles with radius $\varepsilon=0.1$ and $\varepsilon=0.01$ centered in

$$
\mathbf{x}_{1}=\left[\begin{array}{c}
-.5 \\
.5 \\
0
\end{array}\right], \quad \mathbf{x}_{2}=\left[\begin{array}{l}
0 \\
.5 \\
0
\end{array}\right], \quad \mathbf{x}_{3}=\left[\begin{array}{l}
.5 \\
.5 \\
0
\end{array}\right], \quad \mathbf{x}_{4}=\left[\begin{array}{c}
.5 \\
0 \\
0
\end{array}\right], \quad \mathbf{x}_{5}=\left[\begin{array}{c}
.5 \\
-.5 \\
0
\end{array}\right]
$$

In terms of ratio of characteristic length, the ratio $\varepsilon / \lambda_{m}$ is equal to 0.13 in the first configuration and to 0.013 in the second configuration.

For the first configuration, the direct numerical simulation has involved 88073 elements distributed as follows 108 P2-elements, $298 P$-elements, 105 P4-elements, $3640 P 5$-elements and $82969 P 6$-elements. This corresponds to 7183951 degrees of freedom. The time step is approximately $1.6 \times 10^{-4}$. About 25000 time steps are required to reach the final time $t=4$. The duration of the experiment is $10 \mathrm{~min}$ on 576 processors (16 nodes of 36 cores).

The second configuration involves 89751 elements, distributed as follows; $718 P^{1}$-elements, $360 P^{2}$-elements, $221 P^{3}$-elements, $954 P^{4}$-elements, $3579 P^{5}$-elements and $83919 P^{6}$-elements. This corresponds to 7293902 degrees of freedom. The time step is $8.3 \times 10^{-6}$ (about 480000 iterations). The numerical experiments last three hours on 576 processors ( 16 nodes of 36 cores).

It is worth noting here that reducing the diameters of the sphere by a factor 10 only slightly increased the number of elements, since we only need to small elements close from the obstacle. However, this divided the time step by a factor 20 .

The results are depicted for the first configuration in Figure 2. We notice that the numerical solution of the Foldy-Lax model is much closer to the direct numerical solution than the solution of the Born approximation. This is particularly true in the center of the computational domain where a diffusion phenomenon can be observed. This is related to multiscattering which is not correctly taken into account by the Born model.

For the second configuration, the results are depicted in Figure 3. In this case, the radius of the obstacles and consequently the ratio $\varepsilon / \lambda_{m}$ are smaller. Both approximations give excellent qualitative results.

Second numerical experiment: the case of 216 spherical obstacles evenly distributed. We consider here the case of a network of 6 by 6 by 6 spheres with radius $\varepsilon=.01$ whose centers $\mathbf{x}_{i, j, k}$ lie on a regular grid of space step $h=.5$. More precisely, we for $0 \leq i, j, k \leq 5$,

$$
\mathbf{x}_{i, j, k}=[i h-3.5 h, j h-3.5 h, k h-3.5 h]^{T} \text { with } h=.5 .
$$

It is worth noting that we are in the ideal case where the obstacles are clearly small in regards with the wavelength. We thus expect the asymptotic model will deliver very good results when compare with the finite element method. The direct numerical simulation involves 209344 elements, with $32748 P^{1}$-elements, $14994 P^{2}$ elements, $9779 P^{3}$-elements, $9630 P^{4}$-elements, $10911 P^{5}$-elements and $131282 P^{6}$-elements. This corresponds 


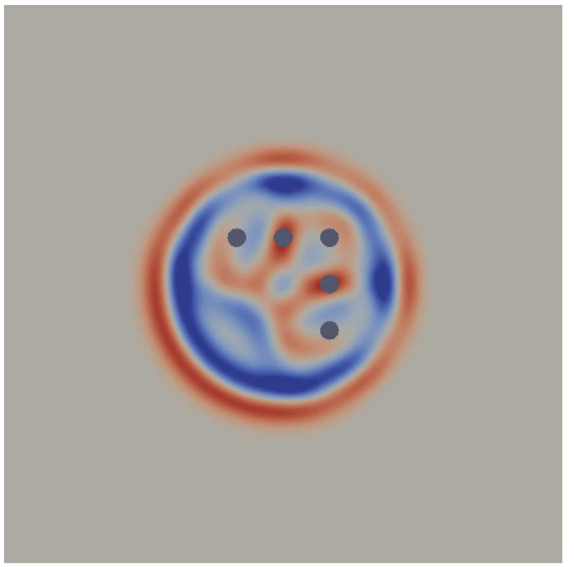

(a)

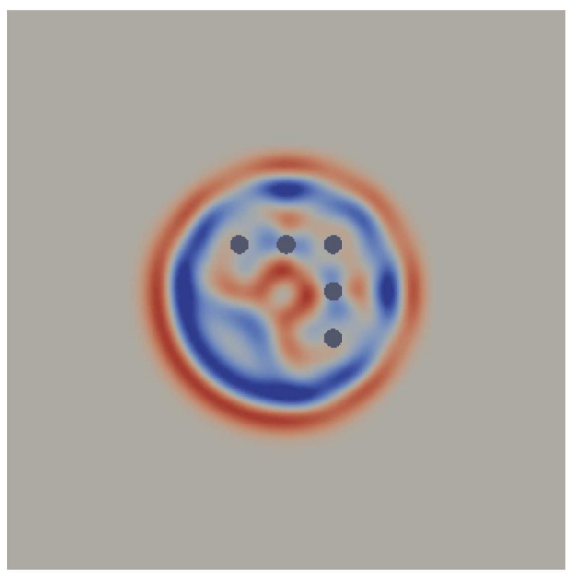

(c)

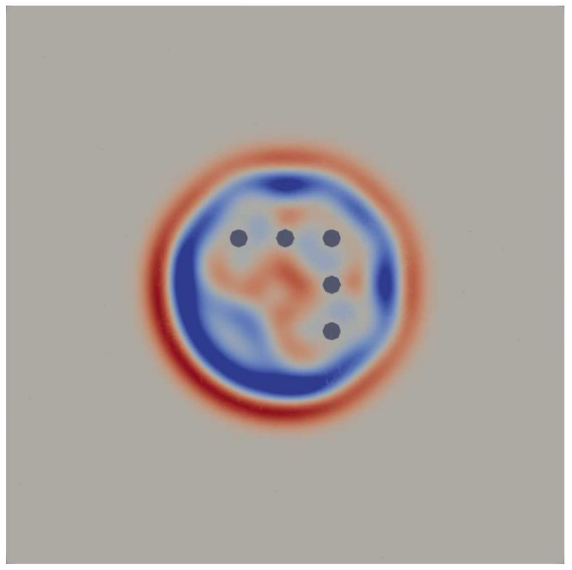

(e)

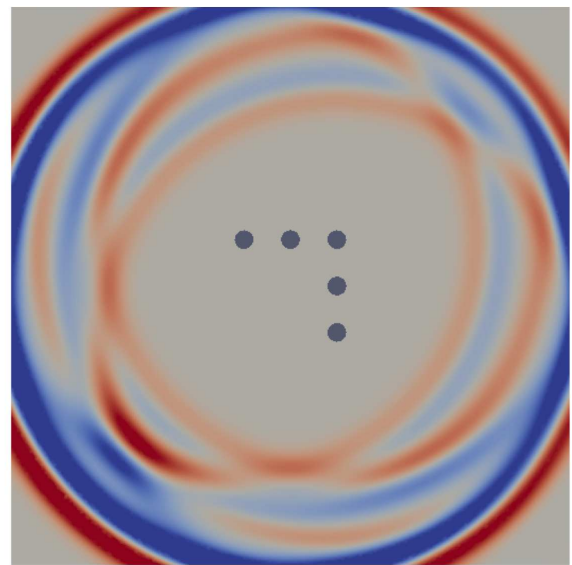

(b)

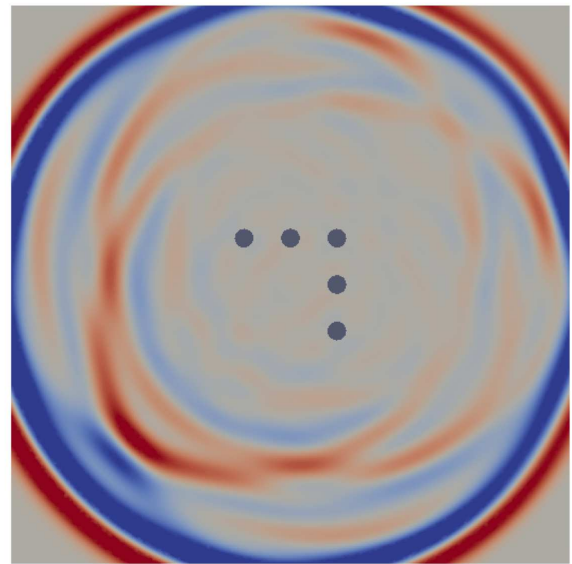

(d)

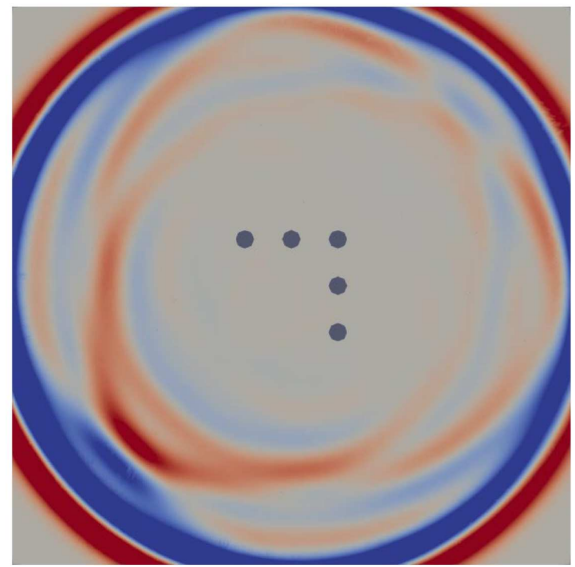

(f)

Figure 2. Comparison between Born, Foldy-Lax and direct numerical simulation. (a) Born $t=2$. (b) Born $t=4$. (c) Foldy $t=2$. (d) Foldy $t=4$. (e) Direct numerical simulation $t=2$. (f) Direct numerical simulation $t=4$. 


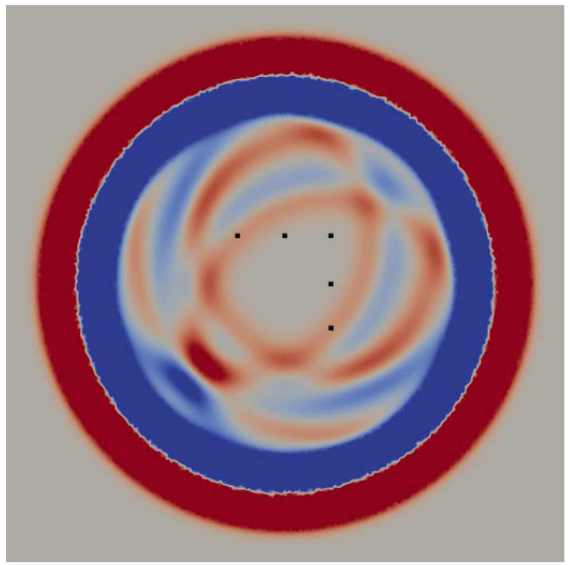

(a)

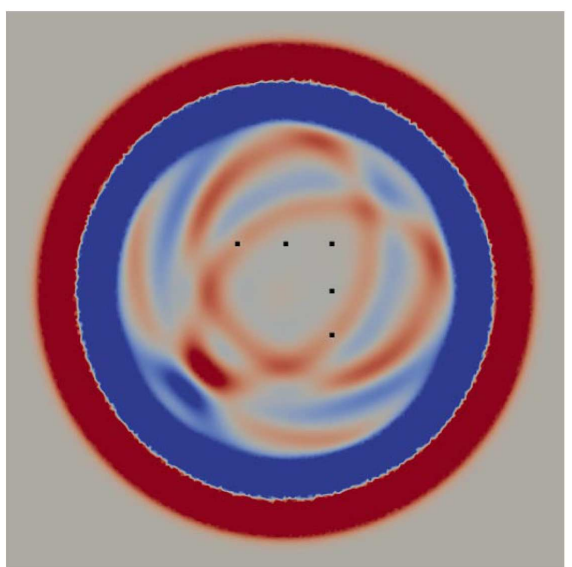

(c)

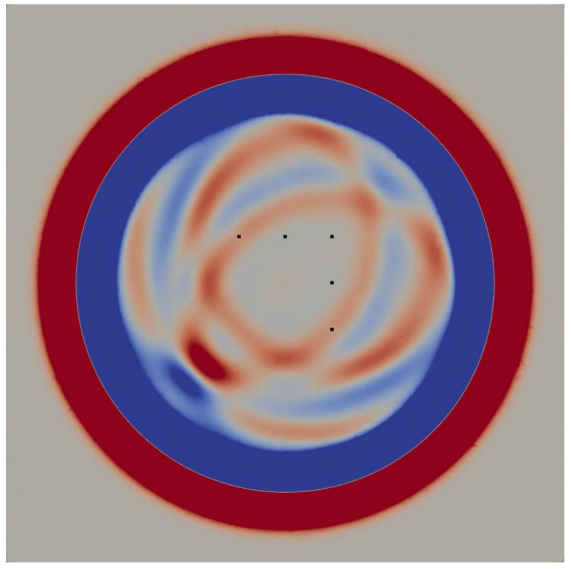

(e)

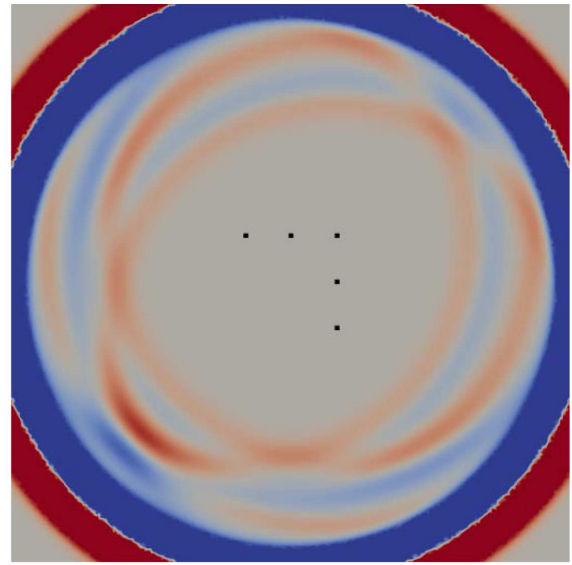

(b)

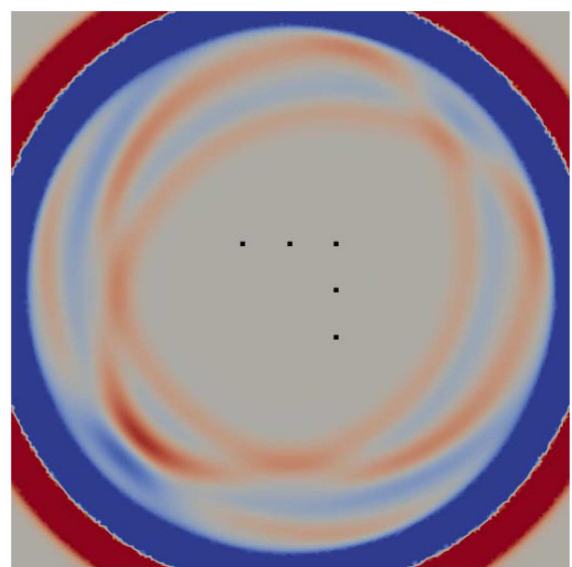

(d)

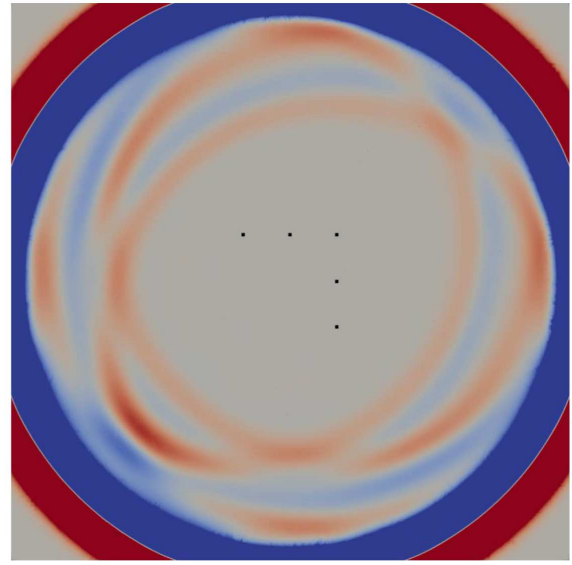

(f)

Figure 3. Comparison between Born, Foldy-Lax and direct numerical simulation. (a) Born $t=3$. (b) Born $t=4$. (c) Foldy $t=3$. (d) Foldy $t=4$. (e) Direct numerical simulation $t=3$. (f) Direct numerical simulation $t=4$. 
to 12452266 degrees of freedom. The time step is $4.62 \times 10^{-6}$. The numerical experiment lasts 12 hours on 576 processors (16 nodes of 36 cores) to compute the solution at time $t=5$.

This case is only affordable with direct numerical simulation performed on a supercomputer. On a laptop it would have taken almost two years. Since the simulation is performed in time-domain the memory issue is less crucial.

A clear difference can be observed close to the boundary of the computational domain. This is due to the approximate outgoing wave condition which does not filter the reflections completely, so we can see a spurious wave which pollutes the finite element calculation.

The Foldy-Lax model took $2.56 \mathrm{~s}$ to solve the problem on a personal computer $(1.8 \mathrm{GHz}$ simple core with $4 \mathrm{~Gb}$ of RAM). These preliminary results are presented in Figure 4 . We can observe the solution at times $t=2$ and $t=3$. Only 36 spheres are visible since we are representing the solution on the plane $z=0.25$.

It is worth mentioning that thanks to Foldy-Lax model, we have been able to perform simulations of acoustic wave diffraction by dense arrays of small obstacles up to 10000 in number. These simulations could not be carried out with the finite element code.

\section{Mathematical anAlysis}

\subsection{Statement of the result}

In Section 2, we have formally written a matched asymptotic expansion $u_{\varepsilon}$ providing a representation of the scattered field in presence of a small obstacle. In the following, we justify this development by proving that the Taylor expansion of $u_{i}$ in (2.20) converges.

For $i>0$, the terms $u_{i}$ are singular functions and according to (2.12) and (2.13), they are represented by a finite sum (see $[32,33]$ for details). As far as convergence is concerned, we will thus focus on the regular term $u_{0} \in C^{\infty}\left(\mathbb{R}^{3} \times \mathbb{R}\right)$, solution to the wave equation (2.4), given by (2.5) along with (2.9) and (2.10).

The computation of the Taylor expansion of $u_{0}$ can be achieved by decomposing (2.5) in two terms

$$
u_{0}(r, \theta, \varphi, t)=\mathfrak{s}^{N}(\mathbf{x}, t)+\mathfrak{u}^{N}(\mathbf{x}, t)
$$

with a finite sum of functions

$$
\mathfrak{s}^{N}(\mathbf{x}, t)=\sum_{n=0}^{N} \sum_{m=-n}^{n} u_{m, n}(r, t) Z_{m, n}(\theta, \varphi)
$$

and a modal series whose convergence deserves a particular attention

$$
\mathfrak{u}^{N}(\mathbf{x}, t)=\sum_{n=N+1}^{+\infty} \sum_{m=-n}^{n} u_{m, n}(r, t) Z_{m, n}(\theta, \varphi)
$$

We then have

Lemma 4.1. The Taylor expansion of $\mathfrak{u}^{N}$ and $\mathfrak{s}^{N}$ with respect to $r$ at order $N$ is given by

$$
\begin{aligned}
\mathfrak{s}^{N}(\mathbf{x}, t) & =\sum_{p=0}^{N} u_{0}^{(p)}(\theta, \varphi, t) r^{p}+\underset{r \rightarrow 0}{O}\left(r^{N+1}\right) \\
\max _{t \leq T}\left|\mathfrak{u}^{N}(\mathbf{x}, t)\right| & =\underset{r \rightarrow 0}{O}\left(r^{N+1}\right),
\end{aligned}
$$

with

$$
u_{0}^{(p)}(\theta, \varphi, t)=\sum_{n=0}^{p} \sum_{m=-n}^{n} \frac{\Lambda_{0, m, n}^{(p+n+1)}(t)}{2 \pi c^{p+n+1}} Q_{n}^{n-p+1}(-1) Z_{m, n}(\theta, \varphi)
$$




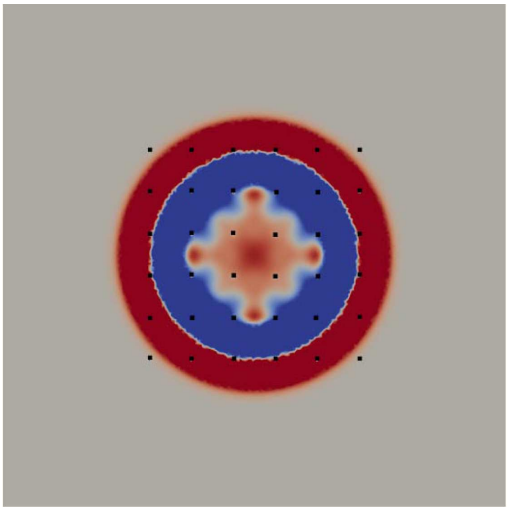

(a)

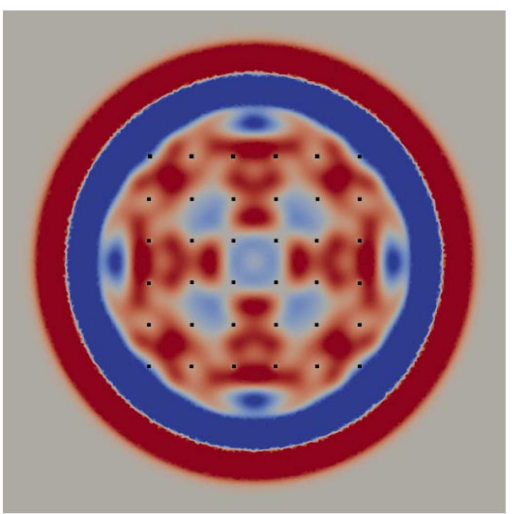

(c)

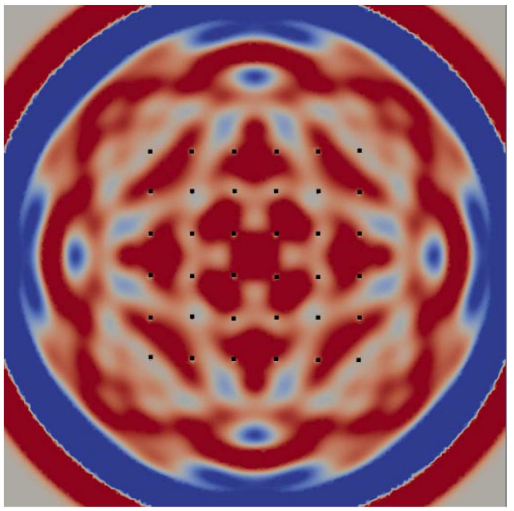

(e)

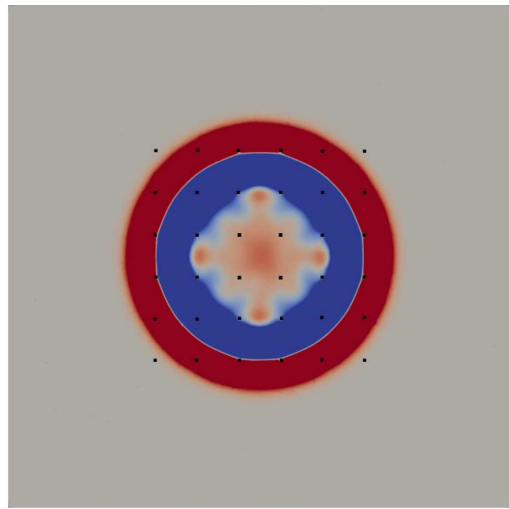

(b)

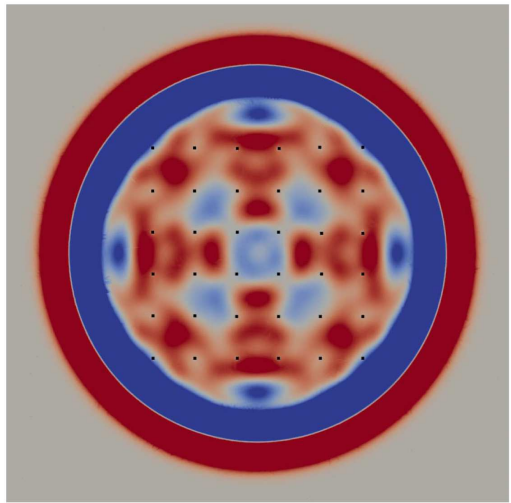

(d)

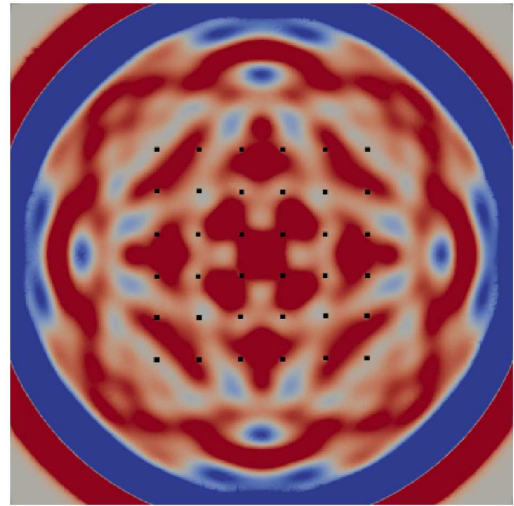

(f)

Figure 4. Comparison between Born, Foldy-Lax and direct numerical simulation. (a) Foldy $t=2$. (b) Direct numerical simulation $t=2$. (c) Foldy $t=3$. (d) direct numerical simulation $t=3$. (e) Foldy $t=4$. (f) Direct numerical simulation $t=4$. 
with

$$
Q_{n}^{k}(X)=\sum_{\ell=0}^{\inf (n, 2 n-k)} q_{n}^{n-\ell} \frac{X^{n-\ell-k}}{(2 n-\ell-k) !} .
$$

It has been proved that $Q_{n}^{k}(-1)=0$ for any odd positive integer number $k$, see [32]. It follows the following Theorem

Theorem 4.2. The Taylor expansion of the regular solution $u_{0}$ is given by

$$
u_{0}(\mathbf{x}, t)=\sum_{p=0}^{N} r^{p} u_{0}^{(p)}(\theta, \varphi, t)+\mathfrak{r}_{0}^{N}(\mathbf{x}, t)
$$

with $u_{0}^{(p)}$ given by $(4.6)$ and the rest $\mathfrak{r}^{N}$

$$
\max _{t \leq T}\left|\mathfrak{r}_{0}^{N}(\mathbf{x}, t)\right|=\underset{r \rightarrow 0}{O}\left(r^{N+1}\right) .
$$

The proof of (4.4) of Lemma 4.1 is simply related to an explicit computation. Details can be found in [32]. The rest of this section is devoted to the proof of (4.5) of Lemma 4.1.

\subsection{Prerequisite for the mathematical analysis}

The key tool for this work is

- the Mellin transform which is part of singularity theory studies, see Section 4.2.1;

- energy estimates for the wave equation, see Section 4.2.2;

- the theory of modal expansion of regular solution to wave equation, see Section 4.2.3.

\subsubsection{Mellin transform: definition and properties}

In this sub-section, we summarize the classic Mellin transformation results, whose proofs have been gathered in [7] (easily accessible online). The reader may also refer to [37].

In what follows, $\beta$ is a real and $p$ is a positive integer. The Kondratiev spaces (see for instance [24], [10] and [14]) are defined by

$$
\left\{\begin{array}{l}
K_{\beta}^{0}=\left\{v: \mathbb{R}^{+} \rightarrow \mathbb{R} \quad \text { such that } \quad r^{-\beta-1 / 2} v(r) \in L^{2}\left(\mathbb{R}^{+}\right)\right\}, \\
K_{\beta}^{p}=\left\{v: \mathbb{R}^{+} \rightarrow \mathbb{R} \quad \text { such that } v^{\{\ell\}} \in K_{\beta}^{0} \quad \forall \ell \leq p\right\},
\end{array}\right.
$$

where the notation $\cdot\{\ell\}$ stands for the differential operator

$$
v^{\{\ell\}}(r)=\left(r \frac{\mathrm{d}}{\mathrm{d} r}\right)^{\ell} v(r)
$$

These spaces are equipped with the Hilbertian norms

$$
\left\{\begin{array}{l}
\|v\|_{K_{\beta}^{0}}=\left(\int_{0}^{+\infty} r^{-2 \beta}|v(r)|^{2} \frac{\mathrm{d} r}{r}\right)^{1 / 2}, \\
\|v\|_{K_{\beta}^{p}}=\left(\sum_{\ell=0}^{p}\left\|v^{\{\ell\}}\right\|_{K_{\beta}^{0}}^{2}\right)^{\frac{1}{2}} .
\end{array}\right.
$$

We denote by $\lambda \in \mathbb{C}$ complex number

$$
\lambda=\beta+i \xi, \quad \beta \in \mathbb{R} \text { and } \xi \in \mathbb{R},
$$

which will serve as the Mellin variable. Let $\mathcal{D}(] 0,+\infty[)$ be the space of functions with compact support included in $] 0,+\infty[$. 
Definition 4.3. For any $v \in \mathcal{D}(] 0,+\infty[)$, the Mellin transform is defined for any $\lambda \in \mathbb{C}$ by

$$
\mathcal{M} v(\lambda)=\frac{1}{\sqrt{2 \pi}} \int_{0}^{+\infty} r^{-\lambda} v(r) \frac{\mathrm{d} r}{r} .
$$

Using the density of $\mathcal{D}(] 0,+\infty[)$ in $K_{\beta}^{p}$, the Mellin transform can be extended to $K_{\beta}^{p}$. Moreover, the Mellin transform $\lambda \mapsto \mathcal{M} v(\lambda)$ is defined for any $v \in K_{\beta}^{p}$ and for any $\lambda \in \mathbb{C}_{\beta}$ where $\mathbb{C}_{\beta}$ denotes the line in the complex plane

$$
\mathbb{C}_{\beta}:=\{\lambda \in \mathbb{C}: \mathcal{R} e(\lambda)=\beta\} .
$$

On the other hand, the Mellin transform is an isomorphism from $K_{\beta}^{p}$ onto $\hat{K}_{\beta}^{p}$, where $\hat{K}_{\beta}^{p}$ stands for

$$
\hat{K}_{\beta}^{p}=\left\{\omega: \mathbb{C}_{\beta} \rightarrow \mathbb{C}: \lambda \mapsto \lambda^{\ell} \omega^{\ell}(\lambda) \in L^{2}\left(\mathbb{C}_{\beta}\right), \quad \forall \ell \leq p\right\} .
$$

In the above definition, $L^{2}\left(\mathbb{C}_{\beta}\right)$ denotes the space of functions on $\mathbb{C}_{\beta}$ equipped with the norm

$$
\|v\|_{L^{2}\left(\mathbb{C}_{\beta}\right)}^{2}=\int_{-\infty}^{+\infty}|v(\beta+i \xi)|^{2} \mathrm{~d} \xi
$$

Property 4.4. Let $p$ be a positive integer. For any $v \in K_{\beta}^{p}$, we have $v^{\{p\}} \in K_{\beta}^{0}$ and for a.e. $\lambda \in \mathbb{C}_{\beta}$

$$
\mathcal{M} v^{\{p\}}(\lambda)=\lambda^{p} \mathcal{M} v(\lambda) .
$$

Property 4.5. Let $v_{q}: \mathbb{R}_{+} \longrightarrow \mathbb{C}$ be defined by $v_{q}(r)=r^{q} v(r)$, with $q \in \mathbb{R}$. If $v \in K_{\beta}^{0}$, then

$$
v_{q} \in K_{\beta+q}^{0} \text {, }
$$

and for any $\lambda \in \mathbb{C}_{q+\beta}$

$$
\mathcal{M} v_{q}(\lambda)=\mathcal{M} v(\lambda-q) .
$$

Proposition 4.6. Let $p$ be a positive integer. For any $v \in K_{\beta_{0}}^{p}$, such that $v(r)=0$ for every $r>\rho_{\star}>0$, the function $v$ belongs to $K_{\beta}^{p}$ for any $\beta \leq \beta_{0}$. The Mellin function is analytical in the half-plane

$$
\mathbb{C}_{]-\infty, \beta_{0}[}:=\left\{\lambda \in \mathbb{C}: \mathcal{R} e(\lambda)<\beta_{0}\right\}
$$

and satisfies for $\operatorname{Re}(\lambda)=\beta<\beta_{0}$

$$
\left|\lambda^{p} \mathcal{M} v(\lambda)\right| \leq \frac{\rho_{\star}^{\beta_{0}-\beta}}{\sqrt{2\left(\beta_{0}-\beta\right)}}\|v\|_{K_{\beta_{0}}^{p}} .
$$

Proposition 4.7. Let $p$ be a positive integer, $\beta_{1}$ and $\beta_{2}$ be two real numbers such that $\beta_{1}<\beta_{2}$. Let $K_{\left[\beta_{1}, \beta_{2}\right]}^{p}:=$ $K_{\beta_{1}}^{p} \cap K_{\beta_{2}}^{p}$. Then for any $\beta \in\left[\beta_{1}, \beta_{2}\right]$, we have $K_{\beta}^{p} \subset K_{\left[\beta_{1}, \beta_{2}\right]}^{p}$.

To prove Theorem 4.2 and thereby estimate the rest $\mathfrak{u}^{N}$, we are going to apply the following theorem coming from singularity theory, that is:

Theorem 4.8. Let $\beta_{0}<\beta_{1}<\beta_{2}$ be three real numbers. Let $v \in K_{\beta_{1}}^{0}$ and $\mathbb{C}_{\left[\beta_{1}, \beta_{2}\right]}$ be the strip of the complex plane defined by

We assume that

$$
\mathbb{C}_{\left[\beta_{1}, \beta_{2}\right]}=\left\{\lambda \in \mathbb{C}: \mathcal{R} e(\lambda) \in\left[\beta_{1}, \beta_{2}\right]\right\}
$$

(i) the Mellin transform $\mathcal{M} v: \mathbb{C}_{\beta_{1}} \rightarrow \mathbb{C}$ admits an analytical continuation $\hat{v}$ in $\mathbb{C}_{\beta_{0}, \beta_{2}[}$;

(ii) there exists $\alpha>0$ such that for any $\lambda=\beta+i \xi \in \mathbb{C}_{\beta_{0}, \beta_{2}[}$ and $|\xi|>1$

$$
\left|\xi^{2} \hat{v}(\lambda)\right| \leq \alpha
$$

then for any $\beta \in] \beta_{0}, \beta_{2}\left[, v \in K_{\beta}^{1}\right.$, and satisfies

$$
|v(r)| \leq \frac{r^{\beta}}{\sqrt{2 \pi}} \int_{-\infty}^{+\infty}|\hat{v}(\beta+i \xi)| \mathrm{d} \xi .
$$




\subsubsection{Energy estimates}

In this paragraph, we recall some elementary properties of the wave equation such as energy conservation or the principle of finite velocity propagation. Let us consider the solution $u_{0} \in C^{\infty}\left(\mathbb{R}^{3} \times \mathbb{R}^{+}\right)$to the Cauchy problem (2.4) defined from a pair of initial data $\left(v_{0}, v_{1}\right)$ satisfying Hypothesis 2.1. The wave field $u_{0}$ propagates with finite speed and its energy is preserved (see [39], Chapt. 2, Sect. 6), that is

$$
E\left(u_{0}, t\right)=E\left(u_{0}, 0\right) \quad \text { with } E\left(u_{0}, t\right)=\int_{\mathbb{R}^{3}}\left|\partial_{t} u_{0}(\mathbf{x}, t)\right|^{2}+c^{2}\left|\nabla u_{0}(\mathbf{x}, t)\right|^{2} \mathrm{~d} \mathbf{x}, \quad \forall t \geq 0 .
$$

From (4.26), we deduce that:

Property 4.9. For any pair of Cauchy data $\left(v_{0}, v_{1}\right)$ satisfying Hypothesis 2.1 , we have for all $t \geq 0$

$$
\left\{\begin{array}{l}
\int_{\mathbb{R}^{3}}\left|\partial_{t}^{2 p+1} u_{0}(\mathbf{x}, t)\right|^{2} \mathrm{~d} \mathbf{x} \leq c^{4 p}\left(\int_{\mathbb{R}^{3}}\left|\Delta^{p} v_{1}(\mathbf{x})\right|^{2}+c^{2} \int_{\mathbb{R}^{3}}\left|\nabla \Delta^{p} v_{0}(\mathbf{x})\right|^{2}\right), \quad \forall p \geq 0, \\
\int_{\mathbb{R}^{3}}\left|\partial_{t}^{2 p} u_{0}(\mathbf{x}, t)\right| \mathrm{d} \mathbf{x} \leq c^{4 p}\left(\int_{\mathbb{R}^{3}}\left|\Delta^{p} v_{0}(\mathbf{x})\right|^{2} \mathrm{~d} \mathbf{x}+\frac{1}{c^{2}} \int_{\mathbb{R}^{3}}\left|\nabla \Delta^{p-1} v_{1}(\mathbf{x})\right|^{2} \mathrm{~d} \mathbf{x}\right), \forall p \geq 1 .
\end{array}\right.
$$

Proof. Since the initial data are in $C^{\infty}$, any time derivative $w=\partial_{t}^{p} u_{0}$ satisfies the wave equation (2.4). For $p \geq 1$, we can thus apply (4.26) to $w$

$$
\int_{\mathbb{R}^{3}}\left|\partial_{t} w(\mathbf{x}, t)\right|^{2} \mathrm{~d} \mathbf{x} \leq E(w, t)=E(w, 0) \quad \forall t \geq 0 .
$$

Property 4.10. For any pair of Cauchy data $\left(v_{0}, v_{1}\right)$ satisfying Hypothesis 2.1 , the support of $u(\cdot, t)$ is enclosed in $B_{r_{*}+c t}$ and

$$
\int_{\mathbb{R}^{3}}\left|u_{0}(\mathbf{x}, t)\right|^{2} \mathrm{~d} \mathbf{x} \leq \frac{\left(r_{\star}+c t\right)^{2}}{c^{2} \pi^{2}}\left(\int_{\mathbb{R}^{3}}\left|v_{1}(\mathbf{x})\right|^{2}+c^{2} \int_{\mathbb{R}^{3}}\left|\nabla v_{0}(\mathbf{x})\right|^{2}\right), \quad \forall t \geq 0
$$

Proof. From the finite speed propagation principle, we have that $u_{0}(\mathbf{x}, t)=0$ except if $|\mathbf{x}| \leq r \leq c t+r_{\star}$. The Poincaré inequality implies that

$$
\left\|u_{0}(\cdot, t)\right\|_{L^{2}\left(B_{r_{\star}+c t}\right)}^{2} \leq \frac{\left(r_{\star}+c t\right)^{2}}{\pi^{2}}\left\|\nabla u_{0}(\cdot, t)\right\|_{L^{2}\left(B_{\left.r_{\star}+c t\right)}\right.}^{2} .
$$

Thus, the previous inequality extends to $R^{3}$ :

$$
\int_{\mathbb{R}^{3}}\left|u_{0}(\mathbf{x}, t)\right|^{2} \mathrm{~d} \mathbf{x} \leq \frac{\left(r_{\star}+c t\right)^{2}}{\pi^{2}} \int_{\mathbb{R}^{3}}\left|\nabla u_{0}(\mathbf{x}, t)\right|^{2} \mathrm{~d} \mathbf{x} .
$$

To complete the proof of Property 4.10, all that remains is to combine (4.26) with (4.31).

\subsubsection{Spectral decomposition}

In what follows, we recall some classical results about separation of variables for writing regular solutions of the wave equation (see e.g. $[17,22,36]$ ).

Any regular solution $u$ of the acoustic wave equation can be decomposed into

$$
u_{0}(\mathbf{x}, t)=\sum_{n=0}^{+\infty} \sum_{m=-n}^{n} u_{m, n}(r, t) \times Z_{m, n}(\theta, \varphi) .
$$


This writing is valid for any value of $t$ and the series converges in $L^{2}\left(\mathbb{R}^{3}\right)$. The terms $u_{m, n}$ are the spectral coefficients of $u_{0}$ in the orthogonal basis $Z_{m, n}$ of $L^{2}(\mathbb{S})$ previously introduced in (2.6); we have:

$$
u_{m, n}(r, t)=\int_{\mathbb{S}} u_{0}(r, \theta, \varphi, t) Z_{m, n}(\theta, \varphi) \sin \theta \mathrm{d} \theta \mathrm{d} \varphi,
$$

where $\mathbb{S}$ denotes the unit sphere parameterized with the angular space variables $(\theta, \varphi)$.

It is then important to list the properties of the functions $Z_{m, n}$. We have:

Property 4.11. The functions $Z_{m, n}$ satisfy

(i) $\left\|Z_{m, n}\right\|_{L^{2}(\mathbb{S})}=1$,

(ii) $Z_{m, n}(0, \varphi)=0, \quad$ if $m \neq 0, \forall \varphi \in[0,2 \pi]$,

(iii) $Z_{0, n}(0, \varphi)=\sqrt{\frac{n+1 / 2}{2 \pi}}$, for $m=0, \forall \varphi \in[0,2 \pi]$.

(iv) $\int_{\mathbb{S}} Z_{m, n}(\theta, \varphi) \sin \theta \mathrm{d} \theta \mathrm{d} \varphi=0$ for $n \neq 0$.

Proof. By construction, the set of functions $Z_{m, n}$ forms an orthonormal basis of $L^{2}(\mathbb{S})$. Indeed according to (2.6), we have

$$
\left\|Z_{m, n}\right\|_{L^{2}(\mathbb{S})}^{2}=\frac{1}{2 \pi} \int_{0}^{2 \pi}|\exp (i m \varphi)|^{2} \mathrm{~d} \varphi \int_{0}^{\pi}\left|\overline{P_{n}^{|m|}}(\cos \theta)\right|^{2} \sin \theta \mathrm{d} \theta .
$$

The corresponding Legendre polynomials are orthogonal and satisfy for any $m \in[0, n]$ (see e.g. [37] p. 37)

$$
\int_{-1}^{1}\left|\overline{P_{n}^{m}}(x)\right|^{2} \mathrm{~d} x=1
$$

By applying the change of variable $x=\cos \theta$, we get the normalized Legendre polynomials which satisfy

$$
\int_{0}^{\pi}\left|\overline{P_{n}^{|m|}}(\cos \theta)\right|^{2} \sin \theta \mathrm{d} \theta=1
$$

This ends the proof of (i).

Still by definition (see $(2.7)), \overline{P_{n}^{m}}(1)=0, \forall m \neq 0$. We thus have (ii) according to (2.6).

By definition (see (2.6) and (2.7)), we have

$$
Z_{0, n}(0,0)=\frac{\overline{P_{n}^{0}}(1)}{\sqrt{2 \pi}}=\sqrt{\frac{n+1 / 2}{2 \pi}},
$$

because $P_{n}(1)=1$. Indeed, following [37] (see p. 35), we have

$$
\left(x^{2}-1\right) P_{n}^{\prime}(x)=n x P_{n}(x)-n P_{n-1}(x),
$$

and by using the recurrence relation (4.38) for $x=1$, we obtain $P_{n}(1)=P_{n-1}(1)$. It is then sufficient to see that $P_{0}(1)=1$ for getting (iii).

By orthogonality, we have for all $n>0$

$$
\int_{\mathbb{S}} Z_{m, n}(\theta, \varphi) Z_{0,0}(\theta, \varphi) \sin (\theta) \mathrm{d} \theta \mathrm{d} \varphi=0
$$

We then remark that $Z_{0,0}(\theta, \varphi)=\frac{1}{\sqrt{4 \pi}}$ which gives (iv) from (4.39). This completes the proof of Property 4.11. 
In [27], the basis functions $Z_{m, n}$ of $L^{2}(\mathbb{S})$ are defined as eigenfunctions of the Laplace-Beltrami operator which reads in spherical coordinates as

$$
\Delta_{\Gamma}=\frac{1}{\sin \theta} \partial_{\theta}\left[\sin \theta \partial_{\theta}\right]+\frac{1}{\sin ^{2} \theta} \partial_{\varphi}^{2}
$$

We actually have

Property 4.12. The functions $Z_{m, n}$ satisfy

$$
\Delta_{\Gamma} Z_{m, n}(\theta, \varphi)=-n(n+1) Z_{m, n}(\theta, \varphi)
$$

The partial differential equation

$$
\Delta u_{0}(\mathbf{x}, t)=\frac{1}{c^{2}} \partial_{t}^{2} u_{0}(\mathbf{x}, t), \quad \text { with } \quad \Delta=\frac{1}{r^{2}} \partial_{r}\left[r^{2} \partial_{r}\right]+\frac{1}{r^{2}} \Delta_{\Gamma}
$$

can be diagonalized by using the spectral coefficients $u_{m, n}$. According to (4.41) together with the fact that the functions $Z_{m, n}$ are orthogonal in $L^{2}(\mathbb{S})$, the coefficients $u_{m, n}$ satisfy for any $r>0$ and $t \geq 0$

$$
\frac{1}{r^{2}} \partial_{r}\left(r^{2} \partial_{r} u_{m, n}(r, t)\right)-\frac{n(n+1)}{r^{2}} u_{m, n}(r, t)=\frac{1}{c^{2}} \partial_{t}^{2} u_{m, n}(r, t)
$$

Each $u_{m, n}$ is a $\mathcal{C}^{\infty}$-function with support in $\left\{(r, t): t \geq 0\right.$ et $\left.0<r<r_{\star}+c t\right\}$. We now rewrite (4.43) as

$$
u_{m, n}^{\{2\}}(r, t)+u_{m, n}^{\{1\}}(r, t)-n(n+1) u_{m, n}(r, t)=\frac{r^{2}}{c^{2}} \partial_{t}^{2} u_{m, n}(r, t) .
$$

with.$\{\ell\}$ defined by (4.11) On the other hand, since for any given time $t$, the function $\mathbf{x} \mapsto u(\mathbf{x}, t)$ belongs to $\mathcal{C}^{\infty}$ and has a compact support, the following energy

$$
E_{\ell}^{T}=\max _{t \leq T} \int_{\mathbb{R}^{3}}\left|\partial_{t}^{\ell} u(r, \theta, \varphi, t)\right|^{2} \mathrm{~d} \mathbf{x} .
$$

is bounded. Thanks to the Parseval equality related to the spectral decomposition, we then have for all $v \in L^{2}(\mathbb{S})$

$$
\int_{0}^{\pi} \int_{0}^{2 \pi}|v(\theta, \varphi)|^{2} \sin \theta \mathrm{d} \theta \mathrm{d} \varphi=\sum_{m, n}\left|v_{m, n}\right|^{2},
$$

with $v_{m, n}=\int_{\mathbb{S}} v(\theta, \varphi) Z_{m, n}(\theta, \varphi) \sin \theta \mathrm{d} \theta \mathrm{d} \varphi$. These terms can be displayed with the spectral coefficients

$$
E_{\ell}^{T}=\max _{t \leq T} \int_{0}^{+\infty} \sum_{m, n}\left|\partial_{t}^{\ell} u_{m, n}(r, t)\right|^{2} r^{2} \mathrm{~d} r
$$

We then have

Proposition 4.13. The space $L^{2}\left(\mathbb{R}^{3}\right)$ can be characterized as

$$
\begin{aligned}
L^{2}\left(\mathbb{R}^{3}\right)= & \left\{v: \mathbb{R}^{3} \rightarrow \mathbb{R} \quad \mid \quad v_{m, n} \in K_{-3 / 2}^{0}, \forall n \geq 0, m \in[-n, n]\right. \\
& \text { and } \left.\sum_{m, n}\left\|v_{m, n}\right\|_{K_{-3 / 2}^{0}}^{2}<+\infty\right\} .
\end{aligned}
$$


Proof. Let $v$ be in $L^{2}\left(\mathbb{R}^{3}\right)$. From (4.46), we know that

$$
\begin{aligned}
\int_{\mathbb{R}^{3}}|v(\mathbf{x})|^{2} \mathrm{~d} \mathbf{x} & =\int_{0}^{+\infty} \int_{0}^{\pi} \int_{0}^{2 \pi}|v(r, \theta, \varphi)|^{2} r^{2} \mathrm{~d} r \sin \theta \mathrm{d} \theta \mathrm{d} \varphi \\
& =\int_{0}^{+\infty} \sum_{m, n}\left|v_{m, n}(r, \theta, \varphi)\right|^{2} r^{2} \mathrm{~d} r .
\end{aligned}
$$

By switching sum and integral terms (following Fubini theorem), it holds that

$$
\int_{\mathbb{R}^{3}}|v(\mathbf{x})|^{2} \mathrm{~d} \mathbf{x}=\sum_{m, n} \int_{0}^{+\infty}\left|v_{m, n}(r, \theta, \varphi)\right|^{2} r^{2} \mathrm{~d} r .
$$

Then, considering that

$$
\int_{0}^{+\infty}\left|v_{m, n}(r, \theta, \varphi)\right|^{2} r^{2} \mathrm{~d} r=\int_{0}^{+\infty}\left|r^{-\left(-\frac{3}{2}\right)} v_{m, n}(r, \theta, \varphi)\right|^{2} \frac{\mathrm{d} r}{r}
$$

the proof of Proposition 4.13 is complete.

\subsection{Convergence proof}

We remind that $\mathfrak{u}^{N}$ is given by the modal expansion (4.3). It is represented by a series to which we want to give meaning. We begin by studying the terms of this series precisely and then we deal with the convergence of this series in the classical sense and not only in $L^{2}\left(\mathbb{R}^{3}\right)$. We follow a two-step approach:

- The first step will consist in estimating precisely the spectral coefficients $u_{m, n}$ for every $n \in \mathbb{N}$ and $-n \leq$ $m \leq n$.

- The second step consists in processing the infinite summation and demonstrate its convergence.

\subsubsection{Control of the terms of the series}

Here we will apply the Theorem 4.8. After testing hypotheses (i) and (ii), we will obtain the behavior close to $r=0$ of $u_{m, n}$ which is described in the following proposition:

Proposition 4.14. Let $N$ be an even integer greater or equal to 2 . For any integer $n \geq N, m \in \mathbb{N}$ with $-n \leq m \leq n$, we have

$$
\left|u_{m, n}(r, t)\right| \leq \frac{1}{n(n+1)} \sqrt{\frac{\pi\left(r_{\star}+c t\right)}{2}} r^{N}\left\|\partial_{T}^{N+2} u_{m, n}\right\|_{K_{-3 / 2}^{0}} \text { with } T=c t .
$$

- Checking (i). The function $\mathbf{x} \mapsto \partial_{t}^{\ell} u(\mathbf{x}, t)$ belongs to $L^{2}\left(\mathbb{R}^{3}\right)$, for every integer $\ell \geq 0$ and $t \geq 0$. The featuring of $L^{2}\left(\mathbb{R}^{3}\right)$, given by Proposition 4.13 , justifies that the spectral coefficients $r \mapsto \partial_{t}^{\ell} u_{m, n}(r, t)$ are functions of $K_{-3 / 2}^{0}$. Since $r \mapsto u_{m, n}(r, t)$ is compactly supported in $\left[0, \rho_{\star}\right]$, with $\rho_{\star}=r_{\star}+c t$, we deduce that

$$
\partial_{t}^{\ell} u_{m, n} \in K_{\beta}^{0}, \quad \forall \ell \in \mathbb{N}, \quad \forall \beta \leq-3 / 2 .
$$

We can thus apply the Mellin transform to equation (4.44) and we use Properties 4.4 and 4.5 to get that for any $t \geq 0$ and for any $\lambda \in \mathbb{C}_{]-\infty,-\frac{3}{2}[}$

$$
\alpha_{n}(\lambda) \mathcal{M} u_{m, n}(\lambda, t)=\partial_{T}^{2} \mathcal{M} u_{m, n}(\lambda-2, t),
$$

with

$$
\alpha_{n}(\lambda)=\lambda^{2}+\lambda-n(n+1) .
$$


We continue by proving that $\lambda \mapsto \mathcal{M} u_{m, n}(\lambda, t)$ admits a meromorphic continuation $\lambda \mapsto \hat{u}_{m, n}(\lambda, t)$ in the complex plane. For $\lambda=\beta+i \xi$ with $\beta<-3 / 2$, the meromorphic continuation $\hat{u}_{m, n}(\lambda, t)$ necessarily coincides with $\mathcal{M} u_{m, n}(\lambda, t)$

$$
\hat{u}_{m, n}(\lambda, t)=\mathcal{M} u_{m, n}(\lambda, t) .
$$

For $\beta \geq-3 / 2$, we construct the meromorphic continuation by means of formula (4.56)

$$
\hat{u}_{m, n}(\lambda, t)=\frac{\partial_{T}^{2 p} \hat{u}_{m, n}\left(\lambda_{p}, t\right)}{\prod_{k=0}^{p-1} \alpha_{n}\left(\lambda_{k}\right)}, \quad \text { with } \lambda_{k}=\lambda-2 k \text { and } \partial_{T}=\partial_{t} / c
$$

where $p$ denotes the integer such that $\beta-2 p \in\left[-\frac{7}{2} ;-\frac{3}{2}\left[\right.\right.$. It is explicitly given by $\left.p=E\left(\frac{7}{4}+\frac{\beta}{2}\right)\right)$ with $\beta=\Re(\lambda)$. The formula (4.59) makes it possible to define $\hat{u}_{m, n}(\cdot, t)$ as a meromorphic function of $\mathbb{C}$.

The zeroes of $\alpha_{n}$ being located at $\lambda=n$ and $\lambda=-n-1$, we can deduce from formula (4.59) that, for all integer $\ell, \lambda \mapsto \partial_{t}^{\ell} \hat{u}_{m, n}(\lambda, t)$ is an analytical function in $\mathbb{C} \backslash\{n+2 k \mid k \in \mathbb{N}\}$.

\section{- Checking (ii).}

Lemma 4.15. For any $\lambda=\beta+i \xi \in \mathbb{C}$ such that $|\xi| \geq 1$

$$
|\xi|^{2}\left|\hat{u}_{m, n}(\lambda, t)\right| \leq \gamma_{n}(t, \beta)
$$

where $\gamma_{n}$ is a function of $\mathbb{R}_{+} \times \mathbb{R} \longrightarrow \mathbb{R}_{+}$locally bounded in .

Proof. We remind that $\lambda=\beta+i \xi$. We first remark that formula (4.59) is also valid for $p=2$

$$
\hat{u}_{m, n}(\lambda, t)=\frac{\partial_{T}^{2 p_{0}} \hat{u}_{m, n}\left(\lambda-2 p_{0}, t\right)}{\prod_{k=0}^{p_{0}-1} \alpha_{n}\left(\lambda_{k}\right)} .
$$

Considering the imaginary part of $\alpha_{n}$, we have

$$
\begin{aligned}
& \text { if } \beta \notin[-1,0] \text { then }\left|\alpha_{n}(\lambda)\right| \geq\left|\mathcal{I} m\left(\alpha_{n}(\lambda)\right)\right|=|(2 \beta+1) \xi| \geq|\xi|, \\
& \text { if } \beta \in[-1,0] \text { then }\left|\alpha_{n}(\lambda)\right| \geq\left|\mathcal{R} e\left(\alpha_{n}(\lambda)\right)\right|=\underbrace{n^{2}+n}_{\geq 0}-\underbrace{\left(\beta^{2}+\beta\right)}_{\leq 0}+\xi^{2} \geq \xi^{2} \geq|\xi| .
\end{aligned}
$$

For $p_{0} \geq 2$ and $|\xi| \geq 1$, it follows

$$
\xi^{2}\left|\hat{u}_{m, n}(\lambda, t)\right| \leq \xi^{p_{0}}\left|\hat{u}_{m, n}(\lambda, t)\right| \leq\left|\partial_{T}^{2 p_{0}} \hat{u}_{m, n}\left(\lambda-2 p_{0}, t\right)\right|
$$

we apply Proposition 4.6 with $\beta_{0}=-3 / 2$, the following estimates hold

- If $\lambda-4<-2(\lambda<2)$,

$$
\left\{\begin{aligned}
\xi^{2}\left|\hat{u}_{m, n}(\lambda, t)\right| & \leq\left|\partial_{T}^{4} \hat{u}_{m, n}(\lambda-4, t)\right| \leq \gamma_{n}^{1}(t, \beta)\left\|\partial_{T}^{4} u_{m, n}(\cdot, t)\right\|_{K_{-3 / 2}^{0}} \\
& \leq \gamma_{n}^{1}(t, \beta)\left\|\partial_{T}^{4} u(\cdot, t)\right\|_{L^{2}\left(\mathbb{R}^{3}\right)} \quad \text { see Prop. 4.13, }
\end{aligned}\right.
$$

with $\gamma_{n}^{1}(t, \beta)=\frac{\left(r_{\star}+c t\right)^{-\frac{3}{2}-\beta+4}}{\sqrt{2\left(-\frac{3}{2}-\beta+4\right)}}$. 
- If $\lambda-4>-2(\lambda>2)$. Let $p_{0}$ be the integer such that $\left.\left.\beta-2 p_{0} \in\right]-4,-2\right]$

$$
\left\{\begin{aligned}
\xi^{2}\left|\hat{u}_{m, n}(\lambda, t)\right| & \leq \xi^{p_{0}}\left|\hat{u}_{m, n}(\lambda, t)\right| \leq\left|\partial_{T}^{2 p_{0}} \hat{u}_{m, n}\left(\lambda-2 p_{0}, t\right)\right| \\
& \leq \gamma_{n}^{2}(t)\left\|\partial_{T}^{2 p_{0}} u_{m, n}(\cdot, t)\right\|_{K_{-3 / 2}^{0}} \leq \gamma_{n}^{2}(t)\left\|\partial_{T}^{2 p_{0}} u(\cdot, t)\right\|_{L^{2}\left(\mathbb{R}^{3}\right)} .
\end{aligned}\right.
$$

with

$$
\gamma_{n}^{2}(t, \beta)=\max _{\beta>2} \frac{\left(r_{\star}+c t\right)^{-\frac{3}{2}-\beta+2 p_{0}(\beta)}}{\sqrt{2\left(-\frac{3}{2}-\beta+2 p_{0}(\beta)\right)}}=\max _{\beta \in[0,2]} \frac{\left(r_{\star}+c t\right)^{\frac{1}{2}+\beta}}{\sqrt{\frac{1}{2}+\beta}} .
$$

The result follows form (4.64) and (4.65).

- Final step. Due to Proposition 4.6, the function $\lambda \mapsto \hat{u}_{m, n}(\lambda, t)$ is analytical for any $\mathcal{R} e(\lambda)<n$ and it satisfies (4.60) for any $|\xi|>1$. Theorem 4.8 implies that $u_{m, n}(\cdot, t) \in K_{\beta}^{1}$ for any $\beta<n$.

Lemma 4.16. Let $n$ and $N$ be two integers with $n>N$ and $N$ even. If $\lambda=N+i \xi$, we have

$$
n(n+1)\left(1+\xi^{2}\right)\left|\hat{u}_{m, n}(\lambda, t)\right| \leq \sqrt{\rho_{\star}}\left\|\partial_{T}^{N+2} u_{m, n}(\cdot, t)\right\|_{K_{-3 / 2}^{0}} .
$$

Proof. If $N$ is even, according to (4.59) for $p=\frac{N}{2}+1$, we have

$$
\hat{u}_{m, n}(\lambda, t)=\frac{\partial_{T}^{N+2} \hat{u}_{m, n}(-2+i \xi, t)}{\alpha_{n}(\lambda)\left(\prod_{k=1}^{N / 2-1} \alpha_{n}\left(\lambda_{k}\right)\right) \alpha_{n}(i \xi)}, \quad \text { with } \lambda_{k}=N-2 k+i \xi .
$$

We remark that for any $\xi \in \mathbb{R}$

$$
\left|\alpha_{n}(i \xi)\right| \geq\left|\mathcal{R} e\left(\alpha_{n}(i \xi)\right)\right|=n^{2}+n+\xi^{2} \geq n(n+1) .
$$

Since $1 \leq k \leq N / 2-1$, it follows that $\beta_{k}:=\mathcal{R} e\left(\lambda_{k}\right)=\beta-2 k \in[2, N-2] \subset[0, N]$. As a consequence, we get

$$
\left|\alpha_{n}\left(\lambda_{k}\right)\right| \geq\left|\mathcal{R} e\left(\alpha_{n}\left(\lambda_{k}\right)\right)\right|=n^{2}+n-\beta_{k}^{2}-\beta_{k}+\xi^{2} \geq n^{2}+n-N^{2}-N+\xi^{2} \geq 1
$$

because $n>N$. Likewise, since $\beta=N$, we have

$$
\left|\alpha_{n}(\lambda)\right| \geq\left|\mathcal{R} e\left(\alpha_{n}(N+i \xi)\right)\right|=n^{2}+n-N^{2}-N+\xi^{2} \geq 1+\xi^{2} .
$$

It follows then from (4.69) to (4.71)

$$
n(n+1)\left(1+\xi^{2}\right)\left|\hat{u}_{m, n}(\lambda, t)\right| \leq\left|\partial_{T}^{N+2} \hat{u}_{m, n}(-2+i \xi, t)\right| .
$$

By applying Proposition 4.6 with $\beta_{0}=-3 / 2$, we get

$$
\left|\partial_{T}^{N+2} \hat{u}_{m, n}(-2+i \xi, t)\right| \leq \sqrt{\rho_{\star}}\left\|\partial_{T}^{N+2} u_{m, n}(\cdot, t)\right\|_{K_{-3 / 2}^{0}} \text {, with } \rho_{\star}=r_{\star}+c t .
$$

Then according to (4.72), Lemma 4.16 is proved.

Finally to get estimate (4.54) and Proposition 4.14, it remains to evaluate the following integral

$$
\int_{-\infty}^{+\infty}\left|\hat{u}_{m, n}(N+i \xi, t)\right| d \xi \leq \frac{\sqrt{\rho_{\star}}}{n(n+1)}(\underbrace{\int_{-\infty}^{+\infty} \frac{\mathrm{d} \xi}{1+\xi^{2}}}_{\pi})\left\|\partial_{T}^{N+2} u_{m, n}(\cdot, t)\right\|_{K_{-3 / 2}^{0}},
$$

and then to apply (4.25) in Theorem 4.8. 


\subsubsection{End of the proof of Theorem 4.2}

Let $\mathbb{S}$ be the unit sphere. We establish preliminary results dealing with the Laplace Beltrami operator.

Lemma 4.17. For any $v \in L^{2}(\mathbb{S})$ such that $\Delta_{\Gamma} v \in L^{2}(\mathbb{S})$ and

$$
\int_{\mathbb{S}} v(\theta, \varphi) \sin \theta \mathrm{d} \theta \mathrm{d} \varphi=0,
$$

we have $v \in L^{\infty}(\mathbb{S})$ and the following estimate holds:

$$
\|v\|_{L^{\infty}(\mathbb{S})} \leq\left\|\Delta_{\Gamma} v\right\|_{L^{2}(\mathbb{S})} .
$$

Proof. We set $\hat{\mathbf{y}} \in \mathbb{S}$ and we introduce a coordinate change that associates $\hat{\mathbf{z}} \in \mathbb{S}$ to $\hat{\mathbf{x}} \in \mathbb{S}$ with

$$
\left\{\begin{array}{l}
\hat{\mathbf{z}}_{1}=\hat{\mathbf{x}} \cdot \hat{\mathbf{y}}_{\perp, 1} \\
\hat{\mathbf{z}}_{2}=\hat{\mathbf{x}} \cdot \hat{\mathbf{y}}_{\perp, 2} \\
\hat{\mathbf{z}}_{3}=\hat{\mathbf{x}} \cdot \hat{\mathbf{y}}
\end{array}\right.
$$

where $\hat{\mathbf{y}}_{\perp, 1}$ and $\hat{\mathbf{y}}_{\perp, 2}$ are two vectors such that $\left(\hat{\mathbf{y}}, \hat{\mathbf{y}}_{\perp, 1}, \hat{\mathbf{y}}_{\perp, 2}\right)$ forms an orthonormal basis of $\mathbb{R}^{3}$. The application $R_{\hat{\mathbf{y}}}: \mathbb{S} \mapsto \mathbb{S}$ which associates $\hat{\mathbf{x}}$ to $\hat{\mathbf{z}}$ is an isometry. Let $\widetilde{v}: \mathbb{S} \mapsto \mathbb{S}$ be the function defined by

$$
\widetilde{v}(\hat{\mathbf{z}})=v(\hat{\mathbf{x}}), \quad \text { with } \quad \hat{\mathbf{z}}=R_{\hat{\mathbf{y}}}(\hat{\mathbf{x}}) .
$$

We remark that the function $\widetilde{v}$ satisfies

$$
\int_{\mathbb{S}} \widetilde{v} \mathrm{~d} s=\int_{\mathbb{S}} v \mathrm{~d} s=0, \quad\left\|\Delta_{\Gamma} \widetilde{v}\right\|_{L^{2}(\mathbb{S})}=\left\|\Delta_{\Gamma} v\right\|_{L^{2}(\mathbb{S})} \quad \text { and } \quad \widetilde{v}\left(\hat{\mathbf{z}}_{\mathrm{top}}\right)=v(\hat{\mathbf{y}}),
$$

where $\hat{\mathbf{z}}_{\mathrm{top}}=(0,0,1)$ denotes the North pole of the sphere. Using the spectral decomposition of $\Delta_{\Gamma}$, the function $\widetilde{v}$ can be written as

$$
\widetilde{v}=\sum_{m, n} \widetilde{v}_{m, n} Z_{m, n}
$$

with $\widetilde{v}_{m, n}=\int_{\mathbb{S}} \widetilde{v} Z_{m, n} \mathrm{~d} s$. Following Property 4.11, we have $Z_{m, n}(0,0)=0$ for any $m \neq 0$. Moreover, since $Z_{0,0}$ is proportional to the constant function and $\int_{\mathbb{S}} \widetilde{v}=0 \mathrm{~d} s$, we obtain $\widetilde{v}_{0,0}=0$. It follows that

$$
\widetilde{v}\left(\hat{\mathbf{z}}_{\mathrm{top}}\right)=\sum_{n=1}^{+\infty} \widetilde{v}_{0, n} Z_{0, n}\left(\hat{\mathbf{z}}_{\mathrm{top}}\right)
$$

with $Z_{0, n}\left(\hat{\mathbf{z}}_{\mathrm{top}}\right)=\sqrt{\frac{(n+1 / 2)}{2 \pi}}($ see Property 4.11$)$. We then apply Cauchy Schwarz inequality

$$
\left|\widetilde{v}\left(\hat{\mathbf{z}}_{\mathrm{top}}\right)\right| \leq\left(\sum_{n=1}^{+\infty} \frac{n+1 / 2}{2 \pi n^{2}(n+1)^{2}}\right)^{1 / 2}\left(\sum_{n=1}^{+\infty} n^{2}(n+1)^{2}\left|\widetilde{v}_{0, n}\right|^{2}\right)^{1 / 2} .
$$

Next, we note that

$$
\left\{\begin{array}{l}
\sum_{n=1}^{+\infty} n^{2}(n+1)^{2}\left|\widetilde{v}_{0, n}\right|^{2} \leq \sum_{m, n} n^{2}(n+1)^{2}\left|\widetilde{v}_{m, n}\right|^{2}=\left\|\Delta_{\Gamma} \widetilde{v}\right\|_{L^{2}(\mathbb{S})}^{2}, \\
\left(\sum_{n>0} \frac{n+1 / 2}{2 \pi n^{2}(n+1)^{2}}\right)^{1 / 2} \leq 1,
\end{array}\right.
$$

and we conclude that

$$
\left|\widetilde{v}\left(\hat{\mathbf{z}}_{\text {top }}\right)\right| \leq\left\|\Delta_{\Gamma} \widetilde{v}\right\|_{L^{2}(\mathbb{S})},
$$

which ends the proof thanks to (4.78). 
Lemma 4.18. Let $N \geq 2$ be an even integer. We have

$$
\left\|\Delta_{\Gamma} \mathfrak{u}^{N}(r, \cdot, t)\right\|_{L^{2}(\mathbb{S})} \leq \sqrt{\frac{\pi\left(r_{\star}+c t\right)}{2}} r^{N}\left\|\partial_{T}^{N+2} u\right\|_{L^{2}\left(\mathbb{R}^{3}\right)} .
$$

Proof. According to Parseval equality (4.46), we get

$$
\left\|\Delta_{\Gamma} \mathfrak{u}^{N}(r, \cdot, t)\right\|_{L^{2}(\mathbb{S})}^{2}=\sum_{n=N+1}^{+\infty} \sum_{m=-n}^{n} n^{2}(n+1)^{2}\left|u_{m, n}(r, t)\right|^{2} .
$$

From Proposition 4.14, we deduce that

$$
\left\|\Delta_{\Gamma} \mathfrak{u}^{N}(r, \cdot, t)\right\|_{L^{2}(\mathbb{S})}^{2} \leq \frac{\pi\left(r_{\star}+c t\right)}{2} r^{2 N} \sum_{n=N+1}^{+\infty} \sum_{m=-n}^{n}\left\|\partial_{T}^{N+2} u_{m, n}\right\|_{K_{-3 / 2}^{0}}^{2} .
$$

We apply Parseval theorem once again to get

$$
\sum_{n=N+1}^{+\infty} \sum_{m=-n}^{n}\left\|\partial_{T}^{N+2} u_{m, n}\right\|_{K_{-3 / 2}^{0}}^{2} \leq\left\|\partial_{T}^{N+2} u\right\|_{L^{2}\left(\mathbb{R}^{3}\right)}^{2}
$$

This ends the proof.

Remark 4.19. The norm involved in the right hand side of inequality (4.84) can be bounded by a constant independent of the time thanks to Property 4.9.

The function $\mathfrak{u}^{N}$ is defined as the remainder of the series (2.5)

$$
\mathfrak{u}^{N}(\mathbf{x}, t)=\sum_{n=N+1}^{+\infty} \sum_{m=-n}^{n} u_{m, n}(r, t) Z_{m, n}(\theta, \varphi) .
$$

Due to Property 4.11 and since this series is convergent in $L^{2}(\mathbb{S})$,

$$
\int_{\mathbb{S}} \mathfrak{u}^{N}(r, \theta, \varphi, t) \sin (\theta) \mathrm{d} \theta \mathrm{d} \varphi=\sum_{n=N+1}^{+\infty} \sum_{m=-n}^{n} u_{m, n}(r, t) \int_{\mathbb{S}} Z_{m, n}(\theta, \varphi) \sin (\theta) \mathrm{d} \theta \mathrm{d} \varphi=0 .
$$

Then, from Lemma 4.17, we obtain that for every $\mathbf{x}$ on the sphere with radius $r$

$$
\left|\mathfrak{u}^{N}(\mathbf{x}, t)\right| \leq \sqrt{\frac{\pi\left(r_{\star}+c t\right)}{2}} r^{N}\left\|\partial_{T}^{N+2} u\right\|_{L^{2}\left(\mathbb{R}^{3}\right)} .
$$

This estimate holds true for any $r>0$ and we deduce a non-optimal estimate of $\mathfrak{u}^{N}$ for any even integer $N$ set in the following proposition.

Proposition 4.20. For any $T>0$ and for any even integer $N$ with $N \geq 2$, we have

$$
\max _{t \leq T}\left|\mathfrak{u}^{N}(\mathbf{x}, t)\right|=\underset{r \rightarrow 0}{O}\left(r^{N}\right) .
$$

Proof. To get the estimate of Theorem 4.2, we perform an order upgrading. Let $N$ be a given integer and let $P$ be an even integer such that $P>N$. By definition of $\mathfrak{u}^{N}$ (see (4.3)), we have

$$
\mathfrak{u}^{N}(\mathbf{x}, t)=\sum_{n=N+1}^{P} \sum_{m=-n}^{n} u_{m, n}(r, t) Z_{m, n}(\theta, \varphi)+\mathfrak{u}^{P}(\mathbf{x}, t),
$$


where $\mathfrak{u}^{P}$ is defined like $\mathfrak{u}^{N}$, replacing in (4.88) $N$ by $P$. According to Proposition 4.20 we know that

$$
\left\{\begin{array}{l}
\max _{t \leq T}\left|\sum_{n=N+1}^{P} \sum_{m=-n}^{n} u_{m, n}(r, t) Z_{m, n}(\theta, \varphi)\right|=\underset{r \rightarrow 0}{O}\left(r^{N+1}\right), \\
\max _{t \leq T}\left|\mathfrak{u}^{P}(\mathbf{x}, t)\right|=\underset{r \rightarrow 0}{O}\left(r^{P}\right)=\underset{r \rightarrow 0}{O}\left(r^{N+1}\right) .
\end{array}\right.
$$

since Theorem 1.3 page 57 of [32] states that $u_{m, n}(r, t)=\underset{r \rightarrow 0}{O}\left(r^{n}\right)$. It follows that

$$
\max _{t \leq T}\left|\mathfrak{u}^{N}(\mathbf{x}, t)\right|=\underset{r \rightarrow 0}{O}\left(r^{N+1}\right)
$$

This ends the proof of Theorem 4.2.

\section{Conclusion And Perspectives}

We have proposed a new solution methodology for solving 3D multiple scattering problems when the size of the obstacles is small with respect to the characteristic wavelength. This work contains two key results. First, it validates an asymptotic representation of the field diffracted by a small obstacle illuminated by an incident acoustic wave of very large characteristic length in front of the radius of the obstacle. The proof is based on Kondratiev's theory and the extensive use of the Mellin transform. To the best of our knowledge, this result is the first one addressing the case of time-dependent wave problems. Second, this work shows the potential of the proposed asymptotic model to numerically simulate the effects of a large number of small obstacles on an incident wave. The proposed asymptotic method is confronted with an advanced direct simulation method based on discontinuous finite elements, the time integration being performed with a leapfrog scheme. In a rather simple case where the number of obstacles is limited to 5 , the asymptotic method is validated by comparison with the finite element method. Then we treat the case of 216 obstacles to show that the asymptotic method continues to deliver an accurate solution for a very short computation time while the finite element method reaches its limits. It would also be interesting to consider the case of penetrable obstacles. A more complicated case would be to treat the case of elastoacoustic interactions in order to quickly detect small defects in large structures. It would also be of great interest to compare our solution methodology with those involving highorder radiation conditions $[1,41]$ and to investigate possible extensions of [3] in the time domain. Indeed, now that we dispose of an accurate representation of the scattered field, we should be able to construct an OSRC in the time domain. Finally, the method we have proposed can be extended to other wave equations. Recently, it has been developed for Maxwell's equations in harmonic regime [25,26]. The time-dependent case is clearly more technical but it is quite possible, at least when considering low order asymptotic models.

Acknowledgements. Hélène Barucq and Julien Diaz have received funding from the European Union's Horizon 2020 research and innovation programme under the Marie Sklodowska-Curie grant agreement No 777778 (MATHROCKS). Vanessa Mattesi and Sébastien Tordeux have received funding from CERFACS and Nouvelle Aquitaine Region.

\section{REFERENCES}

[1] S. Acosta, High order surface radiation conditions for time-harmonic waves in exterior domains. Comput. Methods Appl. Mech. Eng. 322 (2017) 296-310.

[2] M. Ainsworth, P. Monk and W. Muniz, Dispersive and dissipative properties of discontinuous galerkin finite element methods for the second-order wave equation. J. Sci. Comput. 27 (2006) 5-40.

[3] H. Alzubaidi, X. Antoine and C. Chniti, Formulation and accuracy of on-surface radiation conditions for acoustic multiple scattering problems. Appl. Math. Comput. 277 (2016) 82-100.

[4] C. Baldassari, H. Barucq, H. Calandra and J. Diaz, Numerical performances of a hybrid local-time stepping strategy applied to the reverse time migration. Geophys. Prospect. 59 (2011) 907-919.

[5] C. Baldassari, H. Barucq, H. Calandra, B. Denel and J. Diaz, Performance analysis of a high-order discontinuous galerkin method application to the reverse time migration. Commun. Comput. Phys. 11 (2012) 660-673. 
[6] H. Barucq, R. Djellouli and E. Estecahandy, Efficient dg-like formulation equipped with curved boundary edges for solving elasto-acoustic scattering problems. Int. J. Numer. Methods Eng. 98 (2014) 747-780.

[7] H. Barucq, V. Mattesi and S. Tordeux, The mellin transform. Technical Report RR-8743, INRIA Bordeaux Sud Ouest (2015).

[8] H. Barucq, F. Faucher and H. Pham, Localization of small obstacles from back-scattered data at limited incident angles with full-waveform inversion. J. Comput. Phys. 370 (2018) 1-24.

[9] A. Bendali, P.-H. Cocquet and S. Tordeux, Approximation by multipoles of the multiple acoustic scattering by small obstacles in three dimensions and application to the foldy theory of isotropic scattering. Arch. Ration. Mech. Anal. 219 (2016) $1017-1059$.

[10] N. Burk and G. Lebeau, Annales Scientifiques de I'École Normale Supérieure - Injections de Sobolev Probabilistes et Applications 4 (2013).

[11] M. Cassier and C. Hazard, Multiple scattering of acoustic waves by small sound-soft obstacles in two dimensions: mathematical justification of the foldy-lax model. Wave Motion 50 (2013) 18-28.

[12] D.P. Challa and M. Sini, On the justification of the foldy-lax approximation for the acoustic scattering by small rigid bodies of arbitrary shapes. Multiscale Model. Simul. 12 (2014) 55-108.

[13] X. Claeys, Analyse asymptotique et numérique de la diffraction d'ondes par des fils minces. Ph.D. thesis, Université de Versailles Saint-Quentin-en-Yvelines (2008).

[14] M. Costabel and M. Dauge, Les problèmes à coins en 10 leçons.

[15] J.D. De Basabe, M.K. Sen and M.F. Wheeler, The interior penalty discontinuous galerkin method for elastic wave propagation: grid dispersion. Geophys. J. Int. 175 (2008) 83-93.

[16] J. Diaz and M.J. Grote, Multi-level explicit local time-stepping methods for second-order wave equations. Comput. Methods Appl. Mech. Eng. 291 (2015) 240-265.

[17] V.M. Dikasov, An inverse problem for the wave equation in a ball. Funct. Anal. App. 25 (1991) 56-58.

[18] L.L. Foldy, The multiple scattering of waves. I. General theory of isotropic scattering by randomly distributed scatterers. Phys. Rev. 67 (1945) 107.

[19] R.F.B. Frank, W.J. Olver, D.W. Lozier and C.W. Clark, NIST Handbook of Mathematical Functions. NIST and Cambridge University Press (2010).

[20] M.J. Grote, A. Schneebeli and D. Schötzau, Discontinuous galerkin finite element method for the wave equation. SIAM J. Numer. Anal. 44 (2006) 2408-2431.

[21] A. Gumerov and R. Duraiswami, Fast Multipole Methods for the Helmholtz Equation in Three Dimensions. Elsevier (2004).

[22] C. Gundlach, J. Martin-Garcia and D. Garfinkle, Summation by parts methods for spherical harmonic decompositions of the wave equation in any dimensions. Class. Quantum Grav. 30 (2013) 145003.

[23] A.M. Il'in, Matching of Asymptotic Expansions of Solutions of Boundary Value Problems. American Mathematical Society (1991).

[24] V.A. Kondratiev, Boundary value problems for elliptic equations in domains with conical or angular points. Trans. Moscow Math. Soc. 16 (1967) 209-292.

[25] J. Labat, Modélisation multi-échelle de la diffraction des ondes électromagnétiques par de petits obstacles. Ph.D. thesis, Pau (2019).

[26] J. Labat, V. Péron and S. Tordeux, Equivalent multipolar point-source modeling of small spheres for fast and accurate electromagnetic wave scattering computations. Wave Motion 92 (2019) 102409.

[27] N.N. Lebedev, Spherical Functions \& Their Applications. Dover Publication (1975).

[28] J. Li, H. Liu, Z. Shang and H. Sun, Two single-shot methods for locating multiple electromagnetic scatterers. SIAM J. Appl. Math. 73 (2013) 1721-1746.

[29] J. Li, H. Liu and Q. Wang, Locating multiple multiscale electromagnetic scatterers by a single far-field measurement. SIAM J. Imaging Sci. 6 (2013) 2285-2309.

[30] J. Li, H. Liu and J. Zou, Locating multiple multiscale acoustic scatterers. Multiscale Model. Simul. 12 (2014) $927-952$.

[31] P.A. Martin, Multiple Scattering: Interaction of Time-harmonic Waves with N Obstacles. In Vol. 107 of Cambridge University Press. (2006).

[32] V. Mattesi, Propagation des ondes dans un milieu comportant des petites hétérogénéités: analyse asymptotique et calcul numérique. Ph.D. thesis, Université de Pau et des Pays de l'Adour (2014).

[33] V. Mattesi and S. Tordeux, Equivalent source modelling of small heterogeneities in the context of 3D time-domain wave propagation equation. In: Waves 2013. Gammarth, Tunisia (2013).

[34] M. N'Diaye, Étude et développement de méthodes numériques d'ordre élevé pour la résolution des équations différentielles ordinaires (EDO): Applications à la résolution des équations d'ondes acoustiques et électromagnétiques. Ph.D. thesis, 2017. Thèse de doctorat dirigée par Barucq, Hélène et Duruflé, Marc Mathématiques appliquées Pau (2017).

[35] J.-C. Nédélec, Acoustic and electromagnetic equations. In: Vol. 144 of Applied Mathematical Sciences. Integral Representations for Harmonic Problems. Springer-Verlag, New York (2001).

[36] J. Novak, J.-L. Cornou and N. Vasset, A spectral method for the wave equation of divergence-free vectors and symmetric tensors inside a sphere. J. Comput. Phys. 229 (2010) 399-414.

[37] A.D. Poularikas, Transforms and Applications Handbook. CRC Press (2010).

[38] M. Rietmann, M. Grote, D. Peter and O. Schenk, Newmark local time stepping on high-performance computing architectures. J. Comput. Phys. 334 (2017) 308-326. 
[39] M.E. Taylor, Partial differential equations I. Basic theory, 2nd edition. In: Vol. 115 of Applied Mathematical Sciences. Springer, New York (2011).

[40] S. Tordeux, Méthodes asymptotiques pour la propagation des ondes dans les milieux comportant des fentes. Ph.D. thesis, Université de Versailles Saint-Quentin-en-Yvelines (2004).

[41] V. Villamizar, S. Acosta and B. Dastrup, High order local absorbing boundary conditions for acoustic waves in terms of farfield expansions. J. Comput. Phys. 333 (2017) 331-351.

[42] S.N. Vladimir Maz'ya and B.1 Plamenevskij, Asymptotic Theory of Elliptic Boundary Value Problems in Singularly Perturbed Domains. Birkhäuser (2000). 